\title{
Abdeldjalil Slama • Ahmed Boudaoui \\ Approximate controllability of fractional nonlinear neutral stochastic differential inclusion with nonlocal conditions and infinite delay
}

Received: 15 March 2016 / Accepted: 30 January 2017 / Published online: 21 February 2017

(C) The Author(s) 2017. This article is published with open access at Springerlink.com

\begin{abstract}
In this paper we consider a class of fractional nonlinear neutral stochastic evolution inclusions with nonlocal initial conditions in Hilbert space. Using fractional calculus, stochastic analysis theory, operator semigroups and Bohnenblust-Karlin's fixed point theorem, a new set of sufficient conditions are formulated and proved for the existence of solutions and the approximate controllability of fractional nonlinear stochastic differential inclusions under the assumption that the associated linear part of the system is approximately controllable. An example is provided to illustrate the theory.
\end{abstract}

Mathematics Subject Classification $65 \mathrm{C} 30 \cdot 93 \mathrm{~B} 05 \cdot 34 \mathrm{~K} 40 \cdot 34 \mathrm{~K} 45$

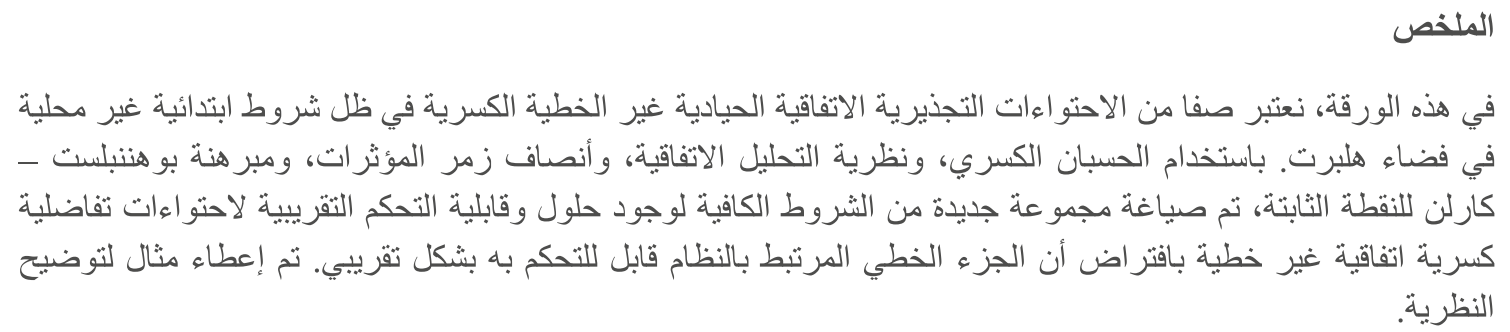

\section{Introduction}

In recent years, the fractional differential equations and inclusions have attracted many physicists, mathematicians and engineers and there was an intensive development of both theory and applications of fractional differential equations ( see $[19,28,30,32,38]$ ).

The fractional differential inclusions, initiated by El-Sayed and Ibrahim [16], arise in the mathematical modeling of certain problems in economics, optimal controls, etc., so the problem of existence of solutions of fractional differential inclusions has been studied by several authors for different kinds of problems and several qualitative results were obtained in $[1,2,11,38,41,42]$.

The problem with nonlocal condition, which is a generalization of the problem of classical condition, was motivated by physical problems. The pioneering work on nonlocal conditions is due to Byszewski (see [810]). Since it is demonstrated that the nonlocal problems have better effects in applications than the classical

A. Slama $(\varangle) \cdot$ A. Boudaoui

Department of Mathematics and Computer Sciences, University of Adrar,

National Road No. 06, Adrar, Algeria

E-mail: slama_dj@yahoo.frr

A. Boudaoui

E-mail: ahmedboudaoui@gmail.com 
Cauchy problems, stochastic differential equations with nonlocal conditions were studied by many authors and some basic results on nonlocal problems have been obtained. Balasubramaniam et al. [3] investigated the approximate controllability of fractional impulsive integro-differential systems with nonlocal conditions in a Hilbert space. Slama and Boudaoui [40] obtained sufficient conditions for the existence of mild solutions for the fractional impulsive stochastic differential equation with nonlocal conditions and infinite delay. For more details see $[4,33,39]$ and the references contained therein.

The controllability is one of the fundamental concepts in linear and nonlinear control theory, and plays a crucial role in both deterministic and stochastic control systems. Moreover, approximate controllable systems are more prevalent and very often approximate controllability is completely adequate in applications (see $[37,38])$. Approximate controllability for semilinear deterministic and stochastic control systems can be found in Mahmudov [26]. Moreover, there are many researchers discussing the approximate controllability for the stochastic fractional systems; for example, see [4,7,33], and the references therein.

For fractional differential inclusions, Sakthivel et al. [38] formulated and proved a new set of sufficient conditions for the approximate controllability of fractional nonlinear differential inclusions. Yan and Jia [43] investigated the existence of mild solutions for a class of impulsive fractional partial neutral functional integrodifferential inclusions with infinite delay and analytic $\alpha$-resolvent operators in Banach spaces. Yan and Jia [44] studied the approximate controllability of partial fractional neutral stochastic functional integro-differential inclusions with state-dependent delay under the assumptions that the corresponding linear system is approximately controllable. Guendouzi and Bousmaha [17] investigated the approximate controllability for a class of fractional neutral stochastic functional integro-differential inclusions involving the Caputo derivative in Hilbert spaces. A new set of sufficient conditions are formulated and proved for the approximate controllability of fractional stochastic integro-differential inclusions under the assumption that the associated linear part of system is approximately controllable.

Recently also, Yan and Lu [45] considered the approximate controllability of a class of fractional stochastic neutral integro-differential inclusions with infinite delay in Hilbert spaces. Sakthivel et al. [39] investigated the approximate controllability of fractional stochastic differential inclusions with nonlocal conditions and established the approximate controllability results for the fractional stochastic control system with infinite delay.

However, to the best of our knowledge, so far no work has been reported in the literature about the existence of solutions and the approximate controllability of fractional nonlinear stochastic differential inclusions with nonlocal conditions and infinite delay of the form (2.1). Inspired by the above mentioned works, the aim of this paper is to fill this gap. The purpose of this paper is to show the existence of solutions and the approximate controllability of fractional nonlinear stochastic differential inclusion of the form (2.1) in a Hilbert space under simple and fundamental assumptions on the system operators, in particular that the corresponding linear system is approximate controllable.

The structure of this paper is as follows: In Sect. 2 we briefly present some basic notations and preliminaries. Section 3 is devoted to the existence of solutions for fractional stochastic control system (2.1). In Sect. 4 we establish the approximate controllability of fractional stochastic control system (2.1). An example to illustrate our results is given in Sect. 5. In the last section, concluding remarks are given.

\section{Preliminaries}

In this section, we introduce some notations and preliminary results, needed to establish our results. Throughout this paper, $\mathbb{H}, \mathbb{U}$ be two separable Hilbert spaces and $L(\mathbb{U}, \mathbb{H})$ be the space of bounded linear operators from $\mathbb{U}$ into $\mathbb{H}$. For convenience, we will use the same notation $\|$. $\|$ to denote the norms in $\mathbb{H}, \mathbb{U}$ and $L(\mathbb{U}, \mathbb{H})$, and use $\langle.,$.$\rangle to denote the inner product of \mathbb{H}$ and $\mathbb{U}$ without any confusion. Let $\left(\Omega, \mathcal{F},\left\{\mathcal{F}_{t}\right\}_{t \geq 0}, \mathbb{P}\right)$ be a complete filtered probability space satisfying that usual conditions (i.e., it is increasing and right continuous, while $\mathcal{F}_{0}$ contains all $\mathbb{P}$-null sets of $\mathcal{F}$ ), and $E($.) denotes the expectation with respect to the measure $\mathbb{P}$. An $\mathbb{H}$-valued random variable is an $\mathcal{F}$-measurable function $x(t): J \rightarrow \mathbb{H}$, and the collection of random variables $S=\{x(t, w): \Omega \rightarrow \mathbb{H} / t \in J\}$ is called a stochastic process. Generally, we just write $x(t)$ instead of $x(t, w)$ and $x(t): J \rightarrow \mathbb{H}$ in the space of $S$. Let $\left\{e_{i}\right\}_{i=1}^{\infty}$ be a complete orthonormal basis of $\mathbb{U}$. Suppose that $W=\left(W_{t}\right)_{t \geq 0}$ is a cylindrical $\mathbb{U}$-valued Wiener process with a finite trace nuclear covariance operator $Q \geq 0$, denote $\operatorname{Tr}(Q)=\sum_{i=1}^{\infty} \lambda_{i}=\lambda<\infty$, which satisfies $Q e_{i}=\lambda_{i} e_{i}$. So, actually, $W(t)=\sum_{i=1}^{\infty} \sqrt{\lambda_{i}} W_{i}(t)$, where $\left\{W_{i}(t)\right\}_{i=1}^{\infty}$ are mutually independent one-dimensional standard Wiener processes. We assume that $\mathcal{F}_{t}=\sigma\{W(s): 0 \leq s \leq t\}$ is the $\sigma$-algebra generated by $W$ and $\mathcal{F}_{T}=\mathcal{F}$. 
Let $L(\mathbb{U}, \mathbb{H})$ denote the space of all bounded linear operator from $\mathbb{U}$ to $\mathbb{H}$. equipped with the usual operator norm $\|$.$\| . For \varphi \in L(\mathbb{U}, \mathbb{H}$ we define

$$
\|\varphi\|_{Q}^{2}=\operatorname{Tr}\left(\varphi Q \varphi^{*}\right)=\sum_{i=1}^{\infty}\left\|\sqrt{\lambda_{i}} \varphi e_{i}\right\|^{2}
$$

If $\|\varphi\|_{Q}^{2}<\infty$, then $\varphi$ is called a $Q-$ Hilbert-Schmidt operator. Let $L_{Q}(\mathbb{U}, \mathbb{H})$ denote the space of all $Q$-Hilbert-Schmidt operator $\varphi$. The completion $L_{Q}(\mathbb{U}, \mathbb{H})$ of $L(\mathbb{U}, \mathbb{H})$ with respect to the topology induced by the norm $\|\cdot\|_{Q}$ where $\|\varphi\|_{Q}^{2}=(\varphi, \varphi)$ is a Hilbert space with the above norm topology.

The collection of all strongly measurable, square integrable, $\mathbb{H}$-valued random variables, denoted by $L_{2}(\Omega, \mathbb{H})$ is a Banach space equipped with the norm $\|x(.)\|_{L_{2}}=\left(E\|x(., \omega)\|_{\mathbb{H}}^{2}\right)^{\frac{1}{2}}$. Let $\mathcal{C}\left(J, L_{2}(\Omega, \mathbb{H})\right)$ be the Banach space of all continuous maps from $J$ into $L_{2}(\Omega, \mathbb{H})$ satisfying $\sup _{0 \leq t \leq T} E\|x(t)\|_{\mathbb{H}}^{2}<\infty . L_{2}^{0}(\Omega, \mathbb{H})$ denote the family of all $\mathcal{F}_{0}$-measurable, $\mathbb{H}$-valued random variables $x(0)$.

The main aim of the present article is to study the approximate controllability of fractional nonlinear stochastic differential inclusions with nonlocal conditions of the form

$$
\left\{\begin{array}{c}
{ }^{c} D_{t}^{\alpha}\left[x(t)-h\left(t, x_{t}\right)\right] \in A x(t)+B u(t)+f\left(t, x_{t}\right) \\
+G\left(t, x_{t}\right) \frac{\mathrm{d} W(t)}{\mathrm{d} t}, \quad t \in J=[0, T], \quad T>0, \\
x(0)+g(x)=x_{0}=\phi, \quad \phi \in \mathcal{B}_{h} .
\end{array}\right.
$$

where $D_{t}^{\alpha}$ is the Caputo fractional derivative of order $\alpha, 0<\alpha<1$, the state variable $x($.) takes the value in the separable Hilbert space $\mathbb{H} ; A: D(A) \subset \mathbb{H} \rightarrow \mathbb{H}$ is the infinitesimal generator of a strongly continuous semigroup of a bounded linear operators $T(t), t \geq 0$ in the Hilbert space $\mathbb{H}$. The history $x_{t}:(-\infty, 0] \rightarrow \mathbb{H}$, $x_{t}(\theta)=x(t+\theta)$, for $t \geq 0$, belongs to an abstract phase space $\mathcal{B}_{h}$. The control function $u($.$) is given in L^{2}(J ; \mathbb{U})$, $\mathbb{U}$ is a Hilbert space; $B$ is a bounded linear operator from $\mathbb{U}$ into $\mathbb{H}$. $h: J \times \mathbb{H} \rightarrow \mathbb{H} ; f: J \times \mathbb{H} \rightarrow \mathbb{H}$, $g: \mathcal{B}_{h} \rightarrow \mathbb{H}$ are appropriate functions to be specified later. $G: J \times \mathbb{H} \rightarrow 2^{\mathbb{H}} \backslash\{\emptyset\}$ is a nonempty bounded, closed and convex multivalued map. The initial data $\phi=\{\phi(t) ; t \in(-\infty, 0]\}$ is an $\mathcal{F}_{0}$-measurable, $\mathcal{B}_{h}$-valued random variable independent of $W(t)$ with finite second moments.

Let $A: \mathcal{D}(A) \subset \mathbb{H} \rightarrow \mathbb{H}$ is the infinitesimal generator of a strongly continuous semigroup of a bounded linear operators $T(t), t \geq 0$ in the Hilbert space $\mathbb{H}$. That is to say, $\|T(t)\| \leq M$ for some constant $M \geq 1$ and every $t \geq 0$. Without loss of generality, we assume that $0 \in \rho(A)$, the resolvent set of $A$. Then it is possible to define the fractional power $A^{\alpha}$ for $0<\alpha \leq 1$, as a closed linear operator on its domain $\mathcal{D}\left(A^{\alpha}\right)$ with inverse $A^{-1}$.

The nonlocal term $g$ has a better effect on the solution and is more precise for physical measurements than the classical condition $x(0)=x_{0}$ alone [38]. For example, $g(x)$ can be written as

$$
g(x)=\sum_{k=1}^{m} c_{k} x\left(t_{k}\right)
$$

where $\left.c_{k}=1 ; 2 ; \ldots ; n\right)$ are given constants and $0<t_{1}<\cdots<t_{n} \leq T$.

Now, we present the abstract space phase $\mathcal{B}_{h}$. Assume that $h:(-\infty, 0] \rightarrow(0,+\infty)$ with $l=\int_{-\infty}^{0} h(t) \mathrm{d} t<$ $\infty$ a continuous function. We define the abstract phase space $\mathcal{B}_{h}$ by

$$
\begin{aligned}
\mathcal{B}_{h}:= & \left\{\phi:(-\infty, 0] \rightarrow \mathbb{H}, \text { for any } a>0,\left(E \mid \phi\left(\left.\theta\right|^{2}\right)^{\frac{1}{2}}\right.\right. \\
& \text { is bounded and measurable function on } \\
& \times[-a, 0] \text { and } \int_{-\infty}^{0} h(s) \sup _{s \leq \theta \leq 0}\left(E \mid \phi\left(\left.\theta\right|^{2}\right)^{\frac{1}{2}}<+\infty\right\} .
\end{aligned}
$$

If $\mathcal{B}_{h}$ is endowed with the norm

$$
\|\phi\|_{\mathcal{B}_{h}}:=\int_{-\infty}^{0} h(s) \sup _{s \leq \theta \leq 0}\left(E|\phi(\theta)|^{2}\right)^{\frac{1}{2}}, \quad \phi \in \mathcal{B}_{h},
$$

then $\left(\mathcal{B}_{h},\|\cdot\|_{\mathcal{B}_{h}}\right)$ is a Banach space $[34,35]$. 
Now we consider the space

$$
\mathcal{B}_{b}:=\left\{x:(-\infty, T] \rightarrow \mathbb{H}, \quad \text { such that }\left.\quad x\right|_{J} \in C(J, \mathbb{H}) \quad x(0)+g(x)=x_{0}=\phi \in \mathcal{B}_{h}\right\}
$$

where $\left.x\right|_{J}$ is the restriction of $x$ to $J$.

We endow a seminorm $\|\cdot\|_{\mathcal{B}_{b}}$ on $\mathcal{B}_{b}$, it is defined by

$$
\|x\|_{\mathcal{B}_{b}}=\|\phi\|_{\mathcal{B}_{h}}+\sup _{0 \leq s \leq T}\left(E\|x(s)\|^{2}\right)^{\frac{1}{2}}, \quad x \in \mathcal{B}_{b} .
$$

We recall the following lemma

Lemma 2.1 [34] Assume that $x \in \mathcal{B}_{b}$; then for $t \in J, x_{t} \in \mathcal{B}_{h}$. Moreover

$$
\left.l\left(E\|x(t)\|^{2}\right)^{\frac{1}{2}} \leq l \sup _{s \in[0, t]} E\|x(s)\|^{2}\right)^{\frac{1}{2}}+\left\|x_{0}\right\|_{\mathcal{B}_{h}}
$$

where $l=\int_{-\infty}^{0} h(s) \mathrm{d} s<\infty$.

The following are basic properties of $A^{\alpha}$ :

(i) $\mathbb{H}_{\alpha}=\mathcal{D}\left(A^{\alpha}\right)$ is a Hilbert space with the norm $\|x\|_{\alpha}=\left\|A^{\alpha} x\right\|$, for $x \in \mathcal{D}\left(A^{\alpha}\right)$.

(ii) $T(t): \mathbb{H} \rightarrow \mathbb{H}_{\alpha}$, for $t \geq 0$.

(iii) $A^{\alpha} T(t) x=T(t) A^{\alpha} x$, for each $x \in \mathcal{D}(A)$ and $t \geq 0$.

(iv) For every $t>0, A^{\alpha} T(t)$ is bounded on $\mathbb{H}$ and there exists $M_{\alpha}>0$ such that

$$
\left\|A^{\alpha} T(t)\right\| \leq \frac{M_{\alpha}}{t^{\alpha}}
$$

(v) $A^{\alpha}$ is a bounded linear operator for $0 \leq \alpha \leq 1$ in $\mathbb{H}$.

Let us recall the following known definition. For more details see $[19,28,30]$.

Definition 2.2 [30] The fractional integral of order $\alpha$ with the lower limit 0 for a function $f$ is defined as

$$
I^{\alpha} f(t)=\frac{1}{\Gamma(\alpha)} \int_{0}^{t}(s-t)^{\alpha-1} f(s) \mathrm{d} s, \quad t>0, \quad \alpha>0
$$

provided the right-hand side is pointwise defined on $[0, \infty)$, where the $\Gamma$ is the gamma function.

Definition 2.3 [12] The Caputo derivative of order $\alpha$ for a function $f:[0, \infty) \rightarrow \mathbb{R}$, which is at least $n$-times differentiable can be defined as

$$
\begin{aligned}
D_{a}^{\alpha} f(t)= & \frac{1}{\Gamma(n-\alpha)} \int_{a}^{t}(t-s)^{n-\alpha-1} f^{(n)}(s) \mathrm{d} s=I_{a}^{(n-\alpha)}\left(\frac{\mathrm{d}^{n} f}{\mathrm{~d} t^{n}}\right)(t), \quad t>0, \\
& 0 \leq n-1<\alpha<n .
\end{aligned}
$$

for $n-1 \leq \alpha<n, n \in \mathbb{N}$. If $0<\alpha \leq 1$, then

$$
D_{a}^{\alpha} f(t)=\frac{1}{\Gamma(1-\alpha)} \int_{a}^{t}(t-s)^{-\alpha}\left(\frac{\mathrm{d} f(s)}{\mathrm{d} s}\right) \mathrm{d} s
$$

Obviously, the Caputo derivative of a constant is equal to zero. The Laplace transform of the Caputo derivative of order $\alpha>0$ is given as

$$
L\left\{D_{t}^{\alpha} f(t) ; \lambda\right\}=\lambda^{\alpha} \widehat{f}(\lambda)-\sum_{k=0}^{n-1} \lambda^{\alpha-k-1} f^{(k)}(0) \quad n-1 \leq \alpha<n .
$$

If $f$ is an abstract function with values in $X$, then integrals which appear in the above definitions are taken in Bochner's sense.

We also introduce some basic definitions and results of multivalued maps. For more details on multivalued maps, we refer to $[5,14,18]$. 
Definition 2.4 A multivalued map $G: \mathbb{H} \rightarrow 2^{\mathbb{H}} \backslash\{\emptyset\}$ is convex (closed) valued if $G(x)$ is convex (closed) for all $x \in \mathbb{H}$. $G$ is bounded on bounded sets if

$$
G(B)=\cup_{x \in B} G(x)
$$

is bounded in $\mathbb{H}$ for any bounded set $B$ of $\mathbb{H}$, that is,

$$
\sup _{x \in B}\{\sup \|y\|: y \in G(x)\}<\infty .
$$

Definition 2.5 $G$ is called upper semicontinuous (u.s.c. for short) on $\mathbb{H}$ if for each $x_{0} \in \mathbb{H}$, the set $G\left(x_{0}\right)$ is a nonempty closed subset of $\mathbb{H}$, and if for each open set $N$ of $\mathbb{H}$ containing $G\left(x_{0}\right)$, there exists an open neighborhood $V$ of $x_{0}$ such that $G(V) \subseteq N$.

Definition 2.6 The multi-valued operator $G$ is called compact if $G(\mathbb{H})$ is a compact subset of $\mathbb{H}$. $G$ is said to be completely continuous if $G(B)$ is relatively compact for every bounded subset $B$ of $\mathbb{H}$.

If the multivalued map $G$ is completely continuous with nonempty values, then $\mathrm{G}$ is u.s.c., if and only if $\mathrm{G}$ has a closed graph, i.e., $x^{n} \rightarrow x^{*}, y^{n} \rightarrow y^{*} ; y^{n} \in G x^{n}$ imply $y^{*} G x^{*}$. $G$ has a fixed point if there is a $x \in \mathbb{H}$ such that $x \in G(x)$.

In the following, $B C C(\mathbb{H})$ denotes the set of all nonempty bounded, closed and convex subset of $\mathbb{H}$.

Definition 2.7 A multi-valued map $G: J \rightarrow B C C(\mathbb{H})$ is said to be measurable if, for each $x \in \mathbb{H}$, the function $\vartheta: J \rightarrow \mathbb{R}$, defined by

$$
\vartheta(t)=d(x, G(t))=\inf \{\|x-z\|: z \in G(t)\}
$$

belong to $L^{1}(J, \mathbb{R})$

Definition 2.8 The multi-valued map $G: J \times \mathbb{H} \rightarrow B C C(H)$ is said to be $L^{2}$-Caratheodory if

(i) $t \mapsto G(t, x)$ is measurable for each $x \in \mathbb{H}$;

(ii) $x \mapsto G(t, x)$ is u.s.c. for almost all $t \in J$;

(iii) for each $r>0$, there exists $l_{r} \in L^{1}(J, \mathbb{R})$ such that

$$
\|G(t, x)\|^{2}:=\sup _{\sigma \in G(t, x)} E\|\sigma\|^{2} \leq l_{r}(t) \text { for all }\|x\|^{2} \leq r \text { and for a.e. } t \in J
$$

Lemma 2.9 [20] Let J be a compact real interval, BCC (HI) be the set of all nonempty, bounded, closed and convex subset of $\mathbb{H}$ and $G$ be a $L^{2}$-Caratheodory multivalued map $S_{G, x} \neq \emptyset$ and let $\Gamma$ be a linear continuous mapping from $L^{2}(J, \mathbb{H})$ to $\mathcal{C}(J, \mathbb{H})$. Then, the operator

$$
\Gamma \circ S_{G}: \mathcal{C}(J, \mathbb{H}) \rightarrow B C C(\mathbb{H}), \quad x \mapsto\left(\Gamma \circ S_{G}\right)(x):=\Gamma\left(S_{G, x}\right),
$$

is a closed graph operator in $\mathcal{C}(J, H) \times \mathcal{C}(J, H)$, where $S_{G, x}$ is known as the selectors set from $G$, is given by

$$
\sigma \in S_{G, x}=\left\{\sigma \in L^{2}(J, L(\mathbb{U}, \mathbb{H})): \sigma(t) \in G(t, x), \text { for a.e.t } \in J\right\}
$$

We present the definition of mild solution for the system (2.1).

Definition 2.10 An $\mathcal{F}_{t}$-adapted stochastic process $x:(-\infty, T] \rightarrow \mathbb{H}$ is called a mild solution of the system (2.1) if $x_{0}=\phi=x(0)+g(x) \in \mathcal{B}_{h}$ on $(-\infty, 0]$ satisfying $x_{0} \in L_{2}^{0}(\Omega, \mathbb{H})$ and the following integral inclusion

$$
\begin{aligned}
x(t) \in & T_{\alpha}(t)[\phi(0)-g(x)-h(0, \phi)]+h\left(t, x_{t}\right)+\int_{0}^{t}(t-s)^{\alpha-1} A S_{\alpha}(t-s) h\left(s, x_{s}\right) \mathrm{d} s \\
& +\int_{0}^{t}(t-s)^{\alpha-1} S_{\alpha}(t-s) B u(s) \mathrm{d} s+\int_{0}^{t}(t-s)^{\alpha-1} S_{\alpha}(t-s) f\left(s, x_{s}\right) \mathrm{d} s \\
& +\int_{0}^{t}(t-s)^{\alpha-1} S_{\alpha}(t-s) G\left(s, x_{s}\right) \mathrm{d} \omega(s), \quad t \in J,
\end{aligned}
$$


is satisfied, where $T_{\alpha}(t)$ and $S_{\alpha}(t)$ are called characteristic solution operators and given by

$$
T_{\alpha}(t)=\int_{0}^{\infty} \xi_{\alpha}(\theta) T\left(t^{\alpha} \theta\right) \mathrm{d} \theta, \quad S_{\alpha}(t)=\alpha \int_{0}^{\infty} \theta \xi_{\alpha}(\theta) T\left(t^{\alpha} \theta\right) \mathrm{d} \theta
$$

and for $\theta \in(0,-\infty)$

$$
\begin{aligned}
& \xi_{\alpha}(\theta)=\frac{1}{\alpha} \theta^{-1-\frac{1}{\alpha}} \varpi_{\alpha}\left(\theta^{-\frac{1}{\alpha}}\right) \geq 0 \\
& \varpi(\theta)=\frac{1}{\pi} \sum_{n=1}^{\infty}(-1)^{n-1} \theta^{-n \alpha-1} \frac{\Gamma(n \alpha+1)}{n !} \sin (n \pi \alpha)
\end{aligned}
$$

$\xi_{\alpha}$ is a probability density function defined on $(0, \infty)$, that is

$$
\xi \alpha(\theta) \geq 0, \theta \in(0, \infty) \text { and } \int_{0}^{\infty} \xi_{\alpha}(\theta) \mathrm{d} \theta=1
$$

Remark 2.11 It is not difficult to verify that for $v \in[0,1]$

$$
\int_{0}^{\infty} \theta^{v} \xi_{\alpha}(\theta) \mathrm{d} \theta=\int_{0}^{\infty} \theta^{-\alpha v} \varpi_{\alpha}(\theta) \mathrm{d} \theta=\frac{\Gamma(1+v)}{\Gamma(1+\alpha v)}
$$

The following lemmas will be used in the proof of our main results.

Lemma 2.12 [46] The operators $T_{\alpha}$ and $S_{\alpha}$ have the following properties:

(i) For any fixed $t \geq 0, T_{\alpha}(t)$ and $S_{\alpha}(t)$ are linear and bounded operators, i.e., for any $x \in \mathbb{H}$,

$$
\left\|T_{\alpha}(t) x\right\| \leq M\|x\|, \quad\left\|S_{\alpha}(t) x\right\| \leq \frac{\alpha M}{\Gamma(1+\alpha)}\|x\| .
$$

(ii) $\left\{T_{\alpha}(t), t \geq 0\right\}$ and $\left\{S_{\alpha}(t), t \geq 0\right\}$ are strongly continuous; which means that for $x \in \mathbb{H}$ and for $0 \leq t^{\prime}<t^{\prime \prime} \leq T$, we have

$$
\left\|T_{\alpha}\left(t^{\prime \prime}\right) x-T_{\alpha}\left(t^{\prime}\right) x\right\| \rightarrow 0 \text { and }\left\|S_{\alpha}\left(t^{\prime \prime}\right) x-S_{\alpha}\left(t^{\prime}\right) x\right\| \rightarrow 0 \text {, as } t^{\prime} \rightarrow t^{\prime \prime} .
$$

(iii) for every $t \geq 0,\left\{T_{\alpha}(t), t \geq 0\right\}$ and $\left\{S_{\alpha}(t), t \geq 0\right\}$ are also compact if $\{T(t), t \geq 0\}$ is compact;

(iv) for any $x \in \mathbb{H}, \beta, \delta \in(0,1)$, we have $A S_{\alpha}(t) x=A^{1-\beta} S_{\alpha}(t) A^{\beta} x$ and

$$
\left\|A^{\delta} S_{\alpha}(t)\right\| \leq \frac{\alpha C_{\delta} \Gamma(2-\delta)}{t^{\alpha \delta} \Gamma(1+\alpha(1-\delta))}, \quad t \in(0, T]
$$

At the end of this section, we recall the fixed point theorem of Bohnenblust and Karlin's ([6]) which is used to establish the existence of the mild solution to the system (2.1).

Lemma 2.13 (Bohnenblust and Karlin [6]) Let D be a nonempty subset of $\mathbb{G}$, which is bounded, closed, and convex. Suppose $G: D \rightarrow 2^{\mathbb{H}} \backslash\{\emptyset\}$ is u.s.c. with closed, convex values, and such that $G(D) \subseteq D$ and $G(D)$ is compact. Then $G$ has a fixed point.

\section{Existence of solutions for fractional stochastic control system}

In this section, we first prove the existence of solutions for fractional control system (2.1) by using BohnenblustKarlin's fixed point theorem. Secondly, we show that under certain assumptions, the approximate controllability of the fractional stochastic inclusion (2.1) is implied by the approximate controllability of the associated linear part (3.1). 
Definition 3.1 Let $x_{T}(\phi, u)$ be the state value of (2.1) at the terminal time $T$ corresponding to the control $u$ and the initial value $\phi$. Introduce the set

$$
\mathcal{R}(T, \phi)=\left\{x_{T}(\phi ; u)(0): u(.) \in L^{2}(J, \mathbb{U})\right\},
$$

which is called the reachable set of (2.1) at the terminal time b and its closure in $\mathbb{H}$ is denoted by $\overline{\mathcal{R}(T, \phi)}$. The system (2.1) is said to be approximately controllable on the interval $J$ if $\overline{\mathcal{R}(T, \phi)}=\mathbb{H}$; that is, given an arbitrary $\epsilon>0$, it is possible to steer from the point $\pi(0)$ to within a distance $\epsilon$ from all points in the state space $\mathbb{H}$ at time $T$.

In order to study the approximate controllability for the fractional control system (2.1), we consider its fractional linear part

$$
\left\{\begin{array}{l}
{ }^{c} D_{t}^{\alpha} x(t) \in A x(t)+B u(t) \quad t \in J=[0, T], \quad T>0 \\
x(0)=\phi, \quad \phi \in B_{h}
\end{array}\right.
$$

It is convenient at this point to introduce the controllability and resolvent operators associated with (3.1) as

$$
\begin{aligned}
L_{0}^{T} & =\int_{0}^{T}(T-s) S_{\alpha}(T-s) B u(s) \mathrm{d} s: \quad L^{2}(J, \mathbb{H}) \rightarrow \mathbb{H} \\
\Gamma_{0}^{T} & =L_{0}^{T}\left(L_{0}^{T}\right)^{*}=\int_{0}^{T}(T-s) S_{\alpha}(T-s) B B^{*} S_{\alpha}^{*}(T-s) \mathrm{d} s: \quad \mathbb{H} \rightarrow \mathbb{H}
\end{aligned}
$$

respectively, where $B^{*}$ denotes the adjoint of $B$ and $S_{\alpha}^{*}(t)$ is the adjoint of $S_{\alpha}(t)$. It is straightforward that the operator $\Gamma_{0}^{T}$ is a linear bounded operator. Let $\mathcal{R}\left(\lambda, \Gamma_{0}^{T}\right)=\left(\lambda I-\Gamma_{0}^{T}\right)^{-1}$ for $\lambda>0$.

Lemma 3.2 [27] The linear fractional control system (3.1) is approximately controllable on [0, T] if and only if $\lambda \mathcal{R}\left(\lambda, \Gamma_{0}^{T}\right) \rightarrow 0$ as $\lambda \longrightarrow 0^{+}$. in the strong operator topology.

In order to establish the existence result, we need the following hypothesis:

(H1) $A$ generates a strongly continuous compact semigroup $T(t): t \geq 0$ in $\mathbb{H}$.

(H2) The function $h: J \times \mathcal{B}_{h} \rightarrow \mathbb{H}$ is continuous and there exists a constant $M_{h}>0,0<\alpha<1$ such that $h$ is $\mathbb{H}_{\alpha}$-valued and

$$
\begin{aligned}
& \left\|A^{\alpha} h\left(t_{1}, x\right)-A^{\alpha} h\left(t_{2}, y\right)\right\|^{2} \leq M_{h}\left(\left|t_{1}-t_{2}\right|+\|x-y\|_{\mathcal{B}_{h}}^{2}\right), \quad x, y \in \mathcal{B}_{h}, \quad t \in J \\
& \left\|A^{\alpha} h(t, x)\right\|^{2} \leq M_{h}\left(1+\|x\|_{\mathcal{B}_{h}}^{2}\right), \quad x \in \mathcal{B}_{h}, \quad t \in J
\end{aligned}
$$

Moreover, there exists a constant $L_{1}>0$ such that the inequality

$$
\left\|A^{\alpha} h(t, x)\right\|^{2} \leq L_{1}
$$

holds for any $x \in \mathcal{B}_{h}$

(H3) The function $f: J \times \mathcal{B}_{h} \rightarrow \mathbb{H}$ is continuous, and there exists a positive constant $M_{f}$ such that the function satisfies that

$$
E\|f(t, x)-f(t, y)\|^{2} \leq M_{f}\|x-y\|_{\mathcal{B}_{h}}^{2}, \quad E\|f(t, x)\|^{2} \leq M_{f}\left(1+\|x\|_{\mathcal{B}_{h}}^{2}\right)
$$

(H4) The multi-valued map $G: J \times \mathcal{B}_{h} \rightarrow B C C\left(L(\mathbb{U}, \mathbb{H})\right.$ is an $L^{2}$-Caratheodory function satisfies the following conditions:

(i) For each $t \in J$, the function $G(t,):. \mathcal{B}_{h} \rightarrow B C C\left(L(\mathbb{U}, \mathbb{H})\right.$ is u.s.c; and for each $x \in \mathcal{B}_{h}$ the function $G(., x)$ is measurable. And for each fixed $x \in \mathcal{B}_{h}$, the set

$$
S_{G, x}=\left\{\sigma \in L^{2}(J, L(\mathbb{U}, \mathbb{H})): \sigma(t) \in G(t, x) \text { a.e. } t \in J\right\}
$$

is nonempty;

(ii) There exists a positive function $l_{r}: J \rightarrow \mathbb{R}^{+}$such that

$$
\sup \left\{E\|\sigma\|^{2}: \sigma(t) \in G(t, x)\right\} \leq l_{r}(t)
$$

for a.e. $t \in J$ and the function $s \mapsto(t-s)^{2(\alpha-1)} l_{r}(s)$ belongs to $L^{1}\left([0, t], \mathbb{R}^{+}\right)$such that

$$
\liminf \frac{\int_{0}^{t}(t-s)^{2(\alpha-1)} l_{r}(s) \mathrm{d} s}{r}=\Lambda<\infty
$$


(H5) $g: C\left([0, T], \mathcal{B}_{h}\right) \rightarrow \mathcal{B}_{h}$ is continuous, and there exists some constant $M_{g}$ such that

$$
E\|g(x)\|^{2} \leq M_{g}\|x\|_{\mathcal{B}_{h}}^{2}
$$

The following lemma is required to define the control function.

Lemma 3.3 [25] For any $\bar{x}_{T} \in L^{2}\left(\mathcal{F}_{T}, H\right)$, there exists $\eta(.) \in L_{\mathcal{F}}^{2}\left(\Omega ; L^{2}\left(J ; L_{2}^{0}\right)\right)$, such that $\bar{x}_{T}=E \bar{x}_{T}+$ $\int_{0}^{T} \eta(s) \mathrm{d} W(s)$.

Now, for any $\lambda>0$ and $\bar{x}_{T} \in L^{2}\left(\mathcal{F}_{T}, H\right)$, we define the control function

$$
\begin{aligned}
u_{x}^{\lambda}(t)= & B^{*} S_{\alpha}^{*}(T-t)\left(\lambda I+\Gamma_{0}^{T}\right)^{-1} \\
& \times\left[E \bar{x}_{T}+\int_{0}^{T} \eta(s) \mathrm{d} W(s)+T_{\alpha}(T)(\phi(0)-g(x)-h(0, \phi))-h\left(T, x_{T}\right)\right] \\
& -B^{*} S_{\alpha}^{*}(T-t) \int_{0}^{T}\left(\lambda I+\Gamma_{s}^{T}\right)^{-1}(T-s)^{\alpha-1} A S_{\alpha}(T-t) h\left(s, x_{s}\right) \mathrm{d} s \\
& -B^{*} S_{\alpha}^{*}(T-t) \int_{0}^{T}\left(\lambda I+\Gamma_{s}^{T}\right)^{-1}(T-s)^{\alpha-1} S_{\alpha}(T-t) f\left(s, x_{s}\right) \mathrm{d} s \\
& -B^{*} S_{\alpha}^{*}(T-t) \int_{0}^{T}\left(\lambda I+\Gamma_{s}^{T}\right)^{-1}(T-s)^{\alpha-1} S_{\alpha}(T-t) \sigma(s) \mathrm{d} W(s)
\end{aligned}
$$

where, $\sigma \in S_{G, x}=\left\{\sigma \in L^{2}(J, L(\mathbb{U}, \mathbb{H})): \sigma(t) \in G(t, x)\right.$ a.e. $\left.t \in J\right\}$.

Let us now explain and prove the following theorem about the existence of solution for the fractional system (2.1).

Theorem 3.4 Assume that the assumptions (H1)-(H5) hold. Then for each $\epsilon>0$, the system (2.1) has a mild solution on $J$ provided that

$$
\begin{aligned}
& {\left[6+\left(\frac{\alpha M M_{B}}{\Gamma(1+\alpha)}\right)^{2} \frac{42 T^{2 \alpha}}{\lambda^{2}}\left(\frac{M M_{B}}{\Gamma(1+\alpha)}\right)^{2}\right]\left[8 M_{g} M^{2} l^{2}+4 M_{h}\left\|A^{-\beta}\right\|^{2} l^{2}+4 \frac{C_{1}(\alpha, \beta) T^{2 \alpha}}{(\alpha \beta)^{2}} M_{h} l^{2}\right.} \\
& \left.\quad+4\left(\frac{M \alpha}{\Gamma(1+\alpha)}\right)^{2} \frac{T^{2 \alpha}}{\alpha^{2}} M_{f} l^{2}+\left(\frac{M \alpha}{\Gamma(1+\alpha)}\right)^{2} \operatorname{Tr}(Q) \Lambda\right]<1
\end{aligned}
$$

Proof In order to prove the existence of mild solutions for system (2.1) transform it into a fixed point problem. For any $\epsilon>0$, we consider the operator $\Psi^{\epsilon}: \mathcal{B}_{b} \rightarrow 2^{\mathcal{B}_{b}}$ defined by $\Psi^{\epsilon} x$ the set of $z \in \mathcal{B}_{b}$ such that

$$
\begin{aligned}
& \Psi x(t)=\phi(t), \quad t \in(-\infty, 0] \\
& z(t)=\left\{\begin{array}{l}
\phi(t) \quad t \in(-\infty, 0] \\
T_{\alpha}(t)(\phi(0)-g(x)-h(0, \phi))+h\left(t, x_{t}\right)+\int_{0}^{t}(t-s)^{\alpha-1} A S_{\alpha}(t-s) h\left(s, x_{s}\right) \mathrm{d} s \\
\left.\quad+\int_{0}^{t}(t-s)^{\alpha-1} S_{\alpha}(t-s) B u_{x}^{\lambda}(s) \mathrm{d} s+\int_{0}^{t}(t-s)^{\alpha-1} S_{\alpha}(t-s) f\left(s, x_{s}\right)\right) \mathrm{d} s \\
+\int_{0}^{t}(t-s)^{\alpha-1} S_{\alpha}(t-s) \sigma(s) \mathrm{d} W(s), t \in J .
\end{array}\right.
\end{aligned}
$$

where $\sigma \in S_{G, x}$.

For $\phi \in \mathcal{B}_{h}$, we define $\widehat{\phi}$ by

$$
\widehat{\phi}(t)= \begin{cases}\phi(t), & t \in(-\infty, 0], \\ T_{\alpha}(t) \phi(0), & t \in J\end{cases}
$$

then $\widehat{\phi} \in \mathcal{B}_{b}$. Let $x(t)=y(t)+\widehat{\phi}(t),-\infty<t<T$. 
It is evident that $y$ satisfies $y_{0}=0, t \in(-\infty, 0]$ and

$$
\begin{aligned}
y(t) \in & T_{\alpha}(t)(-g(y+\widehat{\phi})-h(0, \phi))+h\left(t, y_{t}+\widehat{\phi}_{t}\right) \\
& +\int_{0}^{t}(t-s)^{\alpha-1} A S_{\alpha}(t-s) h\left(s, y_{s}+\widehat{\phi}_{s}\right) \mathrm{d} s \\
& +\int_{0}^{t}(t-s)^{\alpha-1} S_{\alpha}(t-s) B u_{y+\widehat{\phi}}^{\lambda}(s) \mathrm{d} s \\
& +\int_{0}^{t}(t-s)^{\alpha-1} S_{\alpha}(t-s) f\left(s, y_{s}+\widehat{\phi}_{s}\right) \mathrm{d} s \\
& +\int_{0}^{t}(t-s)^{\alpha-1} S_{\alpha}(t-s) G\left(s, y_{s}+\widehat{\phi}_{s}\right) \mathrm{d} W(s), \quad t \in J .
\end{aligned}
$$

where for $\sigma \in S_{G, x}$

$$
\begin{aligned}
u_{y+\widehat{\phi}^{\lambda}}^{\lambda}(t)= & B^{*} S_{\alpha}^{*}(T-t)\left(\lambda I+\Gamma_{0}^{T}\right)^{-1} \\
& \times\left[E \bar{x}_{T}+\int_{0}^{T} \eta(s) \mathrm{d} W(s)+T_{\alpha}(T)(-g(y+\widehat{\phi})-h(0, \phi))-h\left(T, x_{T}\right)\right] \\
& -B^{*} S_{\alpha}^{*}(T-t) \int_{0}^{T}\left(\lambda I+\Gamma_{s}^{T}\right)^{-1}(T-s)^{\alpha-1} A S_{\alpha}(T-t) h\left(s, y_{s}+\widehat{\phi}_{s}\right) \mathrm{d} s \\
& -B^{*} S_{\alpha}^{*}(T-t) \int_{0}^{T}\left(\lambda I+\Gamma_{s}^{T}\right)^{-1}(T-s)^{\alpha-1} S_{\alpha}(T-t) f\left(s, y_{s}+\widehat{\phi}_{s}\right) \mathrm{d} s \\
& -B^{*} S_{\alpha}^{*}(T-t) \int_{0}^{T}\left(\lambda I+\Gamma_{s}^{T}\right)^{-1}(T-s)^{\alpha-1} S_{\alpha}(T-t) \sigma(s) \mathrm{d} W(s)
\end{aligned}
$$

Set $\mathcal{B}_{b}^{0}=\left\{y \in \mathcal{B}_{b}\right.$, such that $\left.y_{0}=0 \in \mathcal{B}_{h}\right\}$ and for any $y \in \mathcal{B}_{b}^{0}$ we have

$$
\|y\|_{\mathcal{B}_{b}^{0}}=\left\|y_{0}\right\|_{\mathcal{B}_{h}}+\sup _{t \in J}\left(E\|y(t)\|^{2}\right)^{\frac{1}{2}}=\sup _{t \in J}\left(E\|y(t)\|^{2}\right)^{\frac{1}{2}}
$$

thus $\left(\mathcal{B}_{b}^{0},\|\cdot\|_{\mathcal{B}_{b}^{0}}\right)$ is a Banach space. Let $\mathcal{B}_{r}=\left\{y \in \mathcal{B}_{b}^{0},\|y\|_{\mathcal{B}_{b}^{0}}^{2} \leq r, r>0\right\}$. The set $\mathcal{B}_{r}$ is clearly a bounded closed convex set in $\mathcal{B}_{b}^{0}$ for each $r>0$ and For each $y \in \mathcal{B}_{r}$. By Lemma 2.1 we have

$$
\begin{aligned}
\left\|y_{t}+\widehat{\phi}_{t}\right\|_{\mathcal{B}_{h}}^{2} \leq & 2\left(\left\|y_{t}\right\|_{\mathcal{B}_{h}}^{2}+\left\|\widehat{\phi}_{t}\right\|_{\mathcal{B}_{h}}^{2}\right) \\
\leq & 4\left(l^{2} \sup _{s \in[0, t]} E\|y(s)\|_{\mathbb{H}}^{2}+\left\|y_{0}\right\|_{\mathcal{B}_{h}}^{2}\right) \\
& +4\left(l^{2} \sup _{s \in[0, t]} E\|\widehat{\phi}(s)\|_{\mathbb{H}}^{2}+\left\|\widehat{\phi}_{0}\right\|_{\mathcal{B}_{h}}^{2}\right) \\
\leq & 4\left(\|\phi\|_{\mathcal{B}_{h}}^{2}+l^{2}\left(r+M^{2} E\|\phi(0)\|_{\mathbb{H}}^{2}\right)\right):=r^{\prime}
\end{aligned}
$$

Define the multi-valued map $\Phi: \mathcal{B}_{r} \rightarrow 2^{\mathcal{B}_{r}}$ by $\Phi y$ the set of $\bar{z} \in \mathcal{B}_{r}$ and there exists $\sigma \in L^{2}(L(\mathbb{U}, \mathbb{H}))$ such that $\sigma \in S_{G, x}$ and

$$
\bar{z}(t)=\left\{\begin{array}{l}
0, t \in(-\infty, 0] \\
T_{\alpha}(t)(-g(y+\widehat{\phi})-h(0, \phi))+h\left(t, y_{t}+\widehat{\phi}_{t}\right) \\
\quad+\int_{0}^{t}(t-s)^{\alpha-1} A S_{\alpha}(t-s) h\left(s, y_{s}+\widehat{\phi}_{s}\right) \mathrm{d} s \\
\quad+\int_{0}^{t}(t-s)^{\alpha-1} S_{\alpha}(t-s) B u_{y+\widehat{\phi}}^{\lambda}(s) \mathrm{d} s \\
+\int_{0}^{t}(t-s)^{\alpha-1} S_{\alpha}(t-s) f\left(s, y_{s}+\widehat{\phi}_{s}\right) \mathrm{d} s \\
+\int_{0}^{t}(t-s)^{\alpha-1} S_{\alpha}(t-s) \sigma(s) \mathrm{d} W(s), \quad t \in J .
\end{array}\right.
$$

Obviously, the operator $\Psi^{\epsilon}$ has a fixed point is equivalent to $\Phi$ has one. So, our aim is to show that $\Phi$ has a fixed point. For the sake of convenience, we subdivide the proof into several steps. 
Step 1: $\Phi$ is convex for each $y \in \mathcal{B}_{r}$. Let if $\bar{z}_{1}, \bar{z}_{2}$ belong to $\Phi y$, then there exist $\sigma_{1}, \sigma_{2} \in S_{G, x}$ such that

$$
\begin{aligned}
\bar{z}_{j}(t)= & T_{\alpha}(t)(-g(y+\widehat{\phi})-h(0, \phi))+h\left(t, y_{t}+\widehat{\phi}_{t}\right) \\
& +\int_{0}^{t}(t-s)^{\alpha-1} A S_{\alpha}(t-s) h\left(s, y_{s}+\widehat{\phi}_{s}\right) \mathrm{d} s+\int_{0}^{t}(t-s)^{\alpha-1} S_{\alpha}(t-s) B u_{y+\widehat{\phi}}^{\lambda}(s) \mathrm{d} s \\
& +\int_{0}^{t}(t-s)^{\alpha-1} S_{\alpha}(t-s) f\left(s, y_{s}+\widehat{\phi}_{s}\right) \mathrm{d} s \\
& +\int_{0}^{t}(t-s)^{\alpha-1} S_{\alpha}(t-s) \sigma_{j}(s) \mathrm{d} W(s), \quad t \in J, \quad j=1,2
\end{aligned}
$$

Let $0 \leq \gamma \leq 1$. Then for each $t \in J$, we have

$$
\begin{aligned}
\left(\gamma \bar{z}_{1}+(1-\gamma) \bar{z}_{2}\right)(t)= & T_{\alpha}(t)(-g(y+\widehat{\phi})-h(0, \phi))+h\left(t, y_{t}+\widehat{\phi}_{t}\right) \\
& +\int_{0}^{t}(t-s)^{\alpha-1} A S_{\alpha}(t-s) h\left(s, y_{s}+\widehat{\phi}_{s}\right) \mathrm{d} s \\
& +\int_{0}^{t}(t-s)^{\alpha-1} S_{\alpha}(t-s) f\left(s, y_{s}+\widehat{\phi}_{s}\right) \mathrm{d} s \\
& +\int_{0}^{t}(t-s)^{\alpha-1} S_{\alpha}(t-s) B B^{*} S_{\alpha}^{*}(T-s)\left\{\left(\lambda I+\Gamma_{0}^{T}\right)^{-1}\right. \\
& \times\left[E \bar{x}_{T}+\int_{0}^{s} \eta(\tau) \mathrm{d} W(\tau)+T_{\alpha}(T)(-g(y+\widehat{\phi})-h(0, \phi))-h\left(T, x_{T}\right)\right] \\
& -\int_{0}^{s}\left(\lambda I+\Gamma_{\tau}^{T}\right)^{-1}(T-\tau)^{\alpha-1} A S_{\alpha}(T-s) h\left(\tau, y_{\tau}+\widehat{\phi}_{\tau}\right) \mathrm{d} \tau \\
& -\int_{0}^{s}\left(\lambda I+\Gamma_{\tau}^{T}\right)^{-1}(T-\tau)^{\alpha-1} S_{\alpha}(T-s) f\left(\tau, y_{\tau}+\widehat{\phi}_{\tau}\right) \mathrm{d} \tau \\
& -\int_{0}^{s}\left(\lambda I+\Gamma_{\tau}^{T}\right)^{-1}(T-\tau)^{\alpha-1} S_{\alpha}(T-s)\left[\gamma \sigma_{1}(\tau)\right. \\
& \left.\left.+(1-\gamma) \sigma_{2}(\tau)\right] \mathrm{d} W(\tau)\right\}(s) \mathrm{d} s \\
& +\int_{0}^{t}(t-s)^{\alpha-1} S_{\alpha}(t-s)\left[\gamma \sigma_{1}(s)+(1-\gamma) \sigma_{2}(s)\right] \mathrm{d} W(s)
\end{aligned}
$$

Since $S_{G, x}$ is convex, $\gamma \bar{z}_{1}+(1-\gamma) \bar{z}_{2} \in S_{G, x}$. Hence $\gamma \bar{z}_{1}+(1-\gamma) \bar{z}_{2} \in \Phi(x)$.

Step 2: We show that there exists some $r>0$ such that $\Phi\left(\mathcal{B}_{r}\right) \subseteq \mathcal{B}_{r}$. If it is not true, then there exists $\lambda>0$ such that for every positive number $r$ and $t \in J$, there exists a function $y^{r}(.) \in \mathcal{B}_{r}$, but $\Phi\left(y^{r}\right) \notin \mathcal{B}_{r}$. that is, $E\left\|\left(\Phi y^{r}\right)(t)\right\|^{2}=\left\{\left\|\bar{z}^{r}\right\|_{\mathcal{B}_{b}}^{2}: \bar{z}^{r} \in \Phi y^{r}\right\} \geq r$. For such $\lambda>0$, we can show that

$$
\begin{aligned}
r \leq & E\left\|\left(\Phi y^{r}\right)(t)\right\|^{2} \\
\leq & 6 E\left\|T_{\alpha}(t)\left(-g\left(y^{r}+\widehat{\phi}\right)-h(0, \phi)\right)\right\|^{2}+6 E\left\|h\left(t, y_{t}^{r}+\widehat{\phi}_{t}\right)\right\|^{2} \\
& +6\left\|\int_{0}^{t}(t-s)^{\alpha-1} A S_{\alpha}(t-s) h\left(s, y_{s}^{r}+\widehat{\phi}_{s}\right) \mathrm{d} s\right\|^{2} \\
& +6 E\left\|\int_{0}^{t}(t-s)^{\alpha-1} S_{\alpha}(t-s) B u_{y^{r}+\widehat{\phi}}^{\lambda}(s) \mathrm{d} s\right\|^{2} \\
& +6 E\left\|\int_{0}^{t}(t-s)^{\alpha-1} S_{\alpha}(t-s) f\left(s, y_{s}^{r}+\widehat{\phi}_{s}\right) \mathrm{d} s\right\|^{2} \\
& +6 E\left\|\int_{0}^{t}(t-s)^{\alpha-1} S_{\alpha}(t-s) \sigma^{r}(s) \mathrm{d} W(s)\right\|^{2}=\sum_{i=1}^{6} I_{i}
\end{aligned}
$$

for some $\sigma^{r} \in S_{G, x}$. 
Let us estimate each term above $I_{i}, i=1, \ldots, 6$. By Lemma 2.1 and assumptions (H1)-(H5), we have

$$
\begin{aligned}
I_{1} & \leq 12 M^{2} M_{g}\left\|y^{r}+\widehat{\phi}\right\|_{\mathcal{B}_{h}}^{2}+12 M^{2}\left\|A^{-\beta}\right\|^{2} M_{h}\left(1+\|\phi\|_{\mathcal{B}_{h}}^{2}\right) \\
& \leq 12 M^{2} M_{g} r^{\prime}+12 M^{2}\left\|A^{-\beta}\right\|^{2} M_{h}\left(1+\|\phi\|_{\mathcal{B}_{h}}^{2}\right) \\
I_{2} & \leq 6\left\|A^{-\beta}\right\|^{2} M_{h}\left(1+\| y_{t}^{r}+\widehat{\phi}_{t}\right) \|_{\mathcal{B}_{h}}^{2} \\
& \leq 6\left\|A^{-\beta}\right\|^{2} M_{h}\left(1+r^{\prime}\right)
\end{aligned}
$$

By a standard calculation involving Lemma 2.12, assumption (H2), Eq. (3.5) and the Holder inequality, we can deduce that

$$
\begin{aligned}
I_{3} & \leq 6 \int_{0}^{t} E\left\|(t-s)^{\alpha-1} A^{1-\beta} S_{\alpha}(t-s) A^{\beta} h\left(s, y_{s}^{r}+\widehat{\phi}_{s}\right) \mathrm{d} s\right\|^{2} \\
& \leq 6 C_{1}(\alpha, \beta) \int_{0}^{t}(t-s)^{\alpha \beta-1} \mathrm{~d} s \int_{0}^{t}(t-s)^{\alpha \beta-1} E \| A^{\beta} h\left(s, y_{s}^{r}+\widehat{\phi}_{s} \|^{2} \mathrm{~d} s\right. \\
& \leq 6 \frac{C_{1}(\alpha, \beta) T^{2 \alpha \beta}}{(\alpha \beta)^{2}} M_{h}\left(1+r^{\prime}\right)
\end{aligned}
$$

where $C_{1}(\alpha, \beta)=\frac{\alpha^{2} C_{1-\beta}^{2} \Gamma^{2}(1+\beta)}{\Gamma^{2}(1+\alpha \beta)}$.

For $I_{4}$, we have

$$
\begin{aligned}
I_{4} & \leq 6 E \int_{0}^{t}\left\|(t-s)^{\alpha-1} S_{\alpha}(t-s) B u_{y^{r}+\widehat{\phi}}^{\lambda}(s)\right\|^{2} \mathrm{~d} s \\
& \leq 6\left(\frac{\alpha M M_{B}}{\Gamma(1+\alpha)}\right)^{2} \frac{T^{\alpha}}{\alpha} \int_{0}^{t}(t-s)^{\alpha-1} E\left\|u_{y^{r}+\widehat{\phi}}^{\lambda}(s)\right\|^{2} \mathrm{~d} s
\end{aligned}
$$

where $\|B\| \leq M_{B}$. By using (H2)-(H5) Holder's inequality, Eq. 3.5 and Lemma 2.12, for some $\sigma^{r} \in S_{G, x}$, we get

$$
\begin{array}{rl}
\left.\| u^{\lambda}\left(s, y^{r}+\widehat{\phi}\right)\right) \|^{2} & 1 \\
\leq & \frac{1}{\lambda^{2}}\left(\frac{\alpha M M_{B}}{\Gamma(1+\alpha)}\right)^{2}\left[7\left\|E \bar{x}_{T}\right\|^{2}+7 E\left\|\int_{0}^{T} \eta(s) \mathrm{d} W(s)\right\|^{2}\right. \\
& \times 7 E\left\|T_{\alpha}(T)\left(-g\left(y^{r}+\widehat{\phi}\right)-h(0, \phi)\right)\right\|^{2}+7 E\left\|h\left(T, y_{T}^{r}+\widehat{\phi}_{T}\right)\right\|^{2} \\
& +7 E\left\|\int_{0}^{T}(T-s)^{\alpha-1} A S_{\alpha}(T-t) h\left(s, y_{s}^{r}+\widehat{\phi}_{s}\right) \mathrm{d} s\right\|^{2} \\
& +7 E\left\|\int_{0}^{T}(T-s)^{\alpha-1} S_{\alpha}(T-t) f\left(s, y_{s}+\widehat{\phi}_{s}\right) \mathrm{d} s\right\|^{2} \\
& \left.+7 E\left\|\int_{0}^{T}(T-s)^{\alpha-1} S_{\alpha}(T-t) \sigma(s) \mathrm{d} W(s)\right\|^{2}\right] \\
\leq & \frac{1}{\lambda^{2}}\left(\frac{\alpha M M_{B}}{\Gamma(1+\alpha)}\right)^{2}\left[7\left\|E \bar{x}_{T}\right\|^{2}+7 \int_{0}^{T} E\|\eta(s)\|_{L_{2}^{0}}^{2} \mathrm{~d} s+14 M^{2} E\left\|g\left(y^{r}+\widehat{\phi}\right)\right\|^{2}\right. \\
& +14 M^{2}\|h(0, \phi)\|^{2}+7 E\left\|h\left(T, y_{T}^{r}+\widehat{\phi}_{T}\right)\right\|^{2} \\
& +7 E\left\|\int_{0}^{T}(T-s)^{\alpha-1} A S_{\alpha}(T-t) h\left(s, y_{s}^{r}+\widehat{\phi}_{s}\right) \mathrm{d} s\right\|^{2} \\
& +7 E\left\|\int_{0}^{T}(T-s)^{\alpha-1} S_{\alpha}(T-t) f\left(s, y_{s}+\widehat{\phi}_{s}\right) \mathrm{d} s\right\|^{2} \\
& \left.+7 E\left\|\int_{0}^{T}(T-s)^{\alpha-1} S_{\alpha}(T-t) \sigma(s) \mathrm{d} W(s)\right\|^{2}\right]
\end{array}
$$




$$
\begin{aligned}
\leq & \frac{7}{\lambda^{2}}\left(\frac{\alpha M M_{B}}{\Gamma(1+\alpha)}\right)^{2}\left[\left\|E \bar{x}_{T}\right\|^{2}+\int_{0}^{T} E\|\eta(s)\|_{\mathscr{L}_{2}^{0}}^{2} \mathrm{~d} s+2 M^{2} M_{g} r^{\prime}\right. \\
& +2 M^{2} M_{h}\left\|A^{-\beta}\right\|^{2}\left(1+\|\phi\|_{\mathcal{B}_{h}}^{2}\right) \\
& +M_{h}\left\|A^{-\beta}\right\|^{2}\left(1+r^{\prime}\right)+\frac{C_{1}(\alpha, \beta) T^{2 \alpha \beta}}{(\alpha \beta)^{2}} M_{h}\left(1+r^{\prime}\right)+\left(\frac{M \alpha}{\Gamma(1+\alpha)}\right)^{2} \frac{T^{2 \alpha}}{\alpha^{2}} M_{f}\left(1+r^{\prime}\right) \\
& \left.+\left(\frac{M \alpha}{\Gamma(1+\alpha)}\right)^{2} \operatorname{Tr}(Q) \int_{0}^{T}(t-s)^{2(\alpha-1)} l_{r}(s) \mathrm{d} s\right]
\end{aligned}
$$

Thus;

$$
I_{4} \leq\left(\frac{\alpha M M_{B}}{\Gamma(1+\alpha)}\right)^{2} \frac{42 T^{2 \alpha}}{\lambda^{2}}\left(\frac{M M_{B}}{\Gamma(1+\alpha)}\right)^{2} \Delta
$$

where

$$
\begin{aligned}
\Delta:= & {\left[\left\|E \bar{x}_{T}\right\|^{2}+\int_{0}^{T} E\|\eta(s)\|_{\mathscr{L}_{2}^{0}}^{2} \mathrm{~d} s+2 M^{2} M_{g} r^{\prime}+2 M^{2} M_{h}\left\|A^{-\beta}\right\|^{2}\left(1+\|\phi\|_{\mathcal{B}_{h}}^{2}\right)\right.} \\
& +M_{h}\left\|A^{-\beta}\right\|^{2}\left(1+r^{\prime}\right)+\frac{C_{1}(\alpha, \beta) T^{2 \alpha \beta}}{(\alpha \beta)^{2}} M_{h}\left(1+r^{\prime}\right)+\left(\frac{M \alpha}{\Gamma(1+\alpha)}\right)^{2} \frac{T^{2 \alpha}}{\alpha^{2}} M_{f}\left(1+r^{\prime}\right) \\
& \left.+\left(\frac{M \alpha}{\Gamma(1+\alpha)}\right)^{2} \operatorname{Tr}(Q) \int_{0}^{T}(t-s)^{2(\alpha-1)} l_{r}(s) \mathrm{d} s\right]
\end{aligned}
$$

Together with assumption (H3) and Eq. (3.5), we have

$$
\begin{aligned}
I_{5} & \leq 6\left(\frac{M \alpha}{\Gamma(1+\alpha)}\right)^{2} \int_{0}^{t}(t-s)^{\alpha-1} \mathrm{~d} s \int_{0}^{t}(t-s)^{\alpha-1} E \| f\left(s, y_{s}^{r}+\widehat{\phi}_{s} \|^{2} \mathrm{~d} s\right. \\
& \leq 6\left(\frac{M \alpha}{\Gamma(1+\alpha)}\right)^{2} \frac{T^{2 \alpha}}{\alpha^{2}} M_{f}\left(1+r^{\prime}\right)
\end{aligned}
$$

A similar argument involves Lemma 2.12, assumption (H4) and Eq. (3.5); we obtain

$$
\begin{aligned}
I_{6} & \leq 6\left(\frac{M \alpha}{\Gamma(1+\alpha)}\right)^{2} \operatorname{Tr}(Q) \int_{0}^{t}(t-s)^{2(\alpha-1)} E\left\|\sigma^{r}(s)\right\|^{2} \mathrm{~d} s \\
& \leq 6\left(\frac{M \alpha}{\Gamma(1+\alpha)}\right)^{2} \operatorname{Tr}(Q) \int_{0}^{t}(t-s)^{2(\alpha-1)} l_{r}(s) \mathrm{d} s
\end{aligned}
$$

Therefore, with these estimates (3.8)-(3.13), (3.7) becomes

$$
\begin{aligned}
r \leq & E\left\|\left(\Phi y^{r}\right)(t)\right\|^{2} \\
\leq & {\left[6+\left(\frac{\alpha M M_{B}}{\Gamma(1+\alpha)}\right)^{2} \frac{42 T^{2 \alpha}}{\lambda^{2}}\left(\frac{M M_{B}}{\Gamma(1+\alpha)}\right)^{2}\right]\left(\left\|E \bar{x}_{T}\right\|^{2}+\int_{0}^{T} E\|\eta(s)\|_{\mathscr{L}^{0}}^{2} \mathrm{~d} s\right) } \\
& +\left[6+\left(\frac{\alpha M M_{B}}{\Gamma(1+\alpha)}\right)^{2} \frac{42 T^{2 \alpha}}{\lambda^{2}}\left(\frac{M M_{B}}{\Gamma(1+\alpha)}\right)^{2}\right]\left[2 M^{2} M_{g} r^{\prime}+2 M^{2} M_{h}\left\|A^{-\beta}\right\|^{2}\left(1+\|\phi\|_{\mathcal{B}_{h}}^{2}\right)\right. \\
& +M_{h}\left\|A^{-\beta}\right\|^{2}\left(1+r^{\prime}\right)+\frac{C_{1}(\alpha, \beta) T^{2 \alpha \beta}}{(\alpha \beta)^{2}} M_{h}\left(1+r^{\prime}\right)+\left(\frac{M \alpha}{\Gamma(1+\alpha)}\right)^{2} \frac{T^{2 \alpha}}{\alpha^{2}} M_{f}\left(1+r^{\prime}\right) \\
& \left.+\left(\frac{M \alpha}{\Gamma(1+\alpha)}\right)^{2} \operatorname{Tr}(Q) \int_{0}^{T}(t-s)^{2(\alpha-1)} l_{r}(s) \mathrm{d} s\right]
\end{aligned}
$$


Dividing both sides of (3.14) by $r$ and taking $r \rightarrow \infty$, we obtain that

$$
\begin{aligned}
& {\left[6+\left(\frac{\alpha M M_{B}}{\Gamma(1+\alpha)}\right)^{2} \frac{42 T^{2 \alpha}}{\lambda^{2}}\left(\frac{M M_{B}}{\Gamma(1+\alpha)}\right)^{2}\right]} \\
& \quad \times\left[8 M_{g} M^{2} l^{2}+4 M_{h}\left\|A^{-\beta}\right\|^{2} l^{2}+4 \frac{C_{1}(\alpha, \beta) T^{2 \alpha}}{(\alpha \beta)^{2}} M_{h} l^{2}\right. \\
& \left.\quad+4\left(\frac{M \alpha}{\Gamma(1+\alpha)}\right)^{2} \frac{T^{2 \alpha}}{\alpha^{2}} M_{f} l^{2}+\left(\frac{M \alpha}{\Gamma(1+\alpha)}\right)^{2} \operatorname{Tr}(Q) \Lambda\right] \geq 1
\end{aligned}
$$

which is a contradiction to our assumption. Thus for $\alpha>0$, for some positive number $r$ and some $\sigma^{r} \in S_{G, x}, \Phi\left(\mathcal{B}_{r}\right) \subseteq \mathcal{B}_{r}$.

Step 3: $\Phi y$ is equicontinuous. Let $\epsilon>0$ small, $0<\epsilon<t<t+h \leq T$. For each $y \in \mathcal{B}_{r}$ and $\bar{z}$ belong to $\Phi y$, there exists $\sigma \in S_{G, x}$ such that for each $t \in J$, we have

$$
\begin{aligned}
& E\|\bar{z}(t+h)-\bar{z}(t)\|^{2} \leq 22\left\|T_{\alpha}(t+h)-T_{\alpha}(t)\right\|^{2}\|(-g(y+\widehat{\phi})-h(0, \phi))\|^{2} \\
& +22 E\left\|h\left(t+h, y_{t+h}+\widehat{\phi}_{t+h}\right)-h\left(t, y_{t}+\widehat{\phi}_{t}\right)\right\|^{2} \\
& +22 E\left\|\int_{t}^{t+h}(t+h-s)^{\alpha-1} A S_{\alpha}(t+h-s) h\left(s, y_{s}+\widehat{\phi}_{s}\right) \mathrm{d} s\right\|^{2} \\
& +22 E\left\|\int_{t}^{t+h}(t+h-s)^{\alpha-1} S_{\alpha}(t+h-s) f\left(s, y_{s}+\widehat{\phi}_{s}\right) \mathrm{d} s\right\|^{2} \\
& +22 E\left\|\int_{t}^{t+h}(t+h-s)^{\alpha-1} S_{\alpha}(t+h-s) \sigma_{j}(s) \mathrm{d} W(s)\right\|^{2} \\
& +22 E\left\|\int_{t}^{t+h}(t+h-s)^{\alpha-1} S_{\alpha}(t+h-s) B u_{y+\widehat{\phi}}^{\lambda}(s) \mathrm{d} s\right\|^{2} \\
& +22 E\left\|\int_{0}^{t-\epsilon}(t+h-s)^{\alpha-1} A\left[S_{\alpha}(t+h-s)-S_{\alpha}(t-s)\right] h\left(s, y_{s}+\widehat{\phi}_{s}\right) \mathrm{d} s\right\| \\
& +22 E\left\|\int_{t-\epsilon}^{t}(t+h-s)^{\alpha-1} A\left[S_{\alpha}(t+h-s)-S_{\alpha}(t-s)\right] h\left(s, y_{s}+\widehat{\phi}_{s}\right) \mathrm{d} s\right\|^{2} \\
& +22 E\left\|\int_{0}^{t-\epsilon}(t+h-s)^{\alpha-1}\left[S_{\alpha}(t+h-s)-S_{\alpha}(t-s)\right] B u_{y+\widehat{\phi}}^{\lambda}(s) \mathrm{d} s\right\|^{2} \\
& +22 E \int_{t-\epsilon}^{t}(t+h-s)^{\alpha-1}\left[S_{\alpha}(t+h-s)-S_{\alpha}(t-s)\right] B u_{y+\widehat{\phi}}^{\lambda}(s) \mathrm{d} s \|^{2} \\
& +22 E\left\|\int_{0}^{t-\epsilon}(t+h-s)^{\alpha-1}\left[S_{\alpha}(t+h-s)-S_{\alpha}(t+s)\right] f\left(s, y_{s}+\widehat{\phi}_{s}\right) \mathrm{d} s\right\|^{2} \\
& +22 E\left\|\int_{t-\epsilon}^{t}(t+h-s)^{\alpha-1}\left[S_{\alpha}(t+h-s)-S_{\alpha}(t-s)\right] f\left(s, y_{s}+\widehat{\phi}_{s}\right) \mathrm{d} s\right\|^{2} \\
& +22 E\left\|\int_{0}^{t-\epsilon}(t+h-s)^{\alpha-1}\left[S_{\alpha}(t+h-s)-S_{\alpha}(t-s)\right] \sigma(s) \mathrm{d} W(s)\right\|^{2} \\
& +22 E\left\|\int_{t-\epsilon}^{t}(t+h-s)^{\alpha-1}\left[S_{\alpha}(t+h-s)-S_{\alpha}(t-s)\right] \sigma(s) \mathrm{d} W(s)\right\|^{2} \\
& +22 E\left\|\int_{0}^{t-\epsilon}\left[(t+h-s)^{\alpha-1}-(t-s)^{\alpha-1}\right] A S_{\alpha}(t-s) h\left(s, y_{s}+\widehat{\phi}_{s}\right) \mathrm{d} s\right\|^{2} \\
& +22 E\left\|\int_{t-\epsilon}^{t}\left[(t+h-s)^{\alpha-1}-(t-s)^{\alpha-1}\right] A S_{\alpha}(t-s) h\left(s, y_{s}+\widehat{\phi}_{s}\right) \mathrm{d} s\right\|^{2}
\end{aligned}
$$




$$
\begin{aligned}
& +22 E\left\|\int_{0}^{t-\epsilon}\left[(t+h-s)^{\alpha-1}-(t-s)^{\alpha-1}\right] S_{\alpha}(t-s) B u_{y+\widehat{\phi}}^{\lambda}(s) \mathrm{d} s\right\|^{2} \\
& +22 E\left\|\int_{t-\epsilon}^{t}\left[(t+h-s)^{\alpha-1}-(t-s)^{\alpha-1}\right] S_{\alpha}(t-s) B u_{y+\widehat{\phi}}^{\lambda}(s) \mathrm{d} s\right\|^{2} \\
& +22 E\left\|\int_{0}^{t-\epsilon}\left[(t+h-s)^{\alpha-1}-(t-s)^{\alpha-1}\right] S_{\alpha}(t-s) f\left(s, y_{s}+\widehat{\phi}_{s}\right) \mathrm{d} s\right\|^{2} \\
& +22 E\left\|\int_{t-\epsilon}^{t}\left[(t+h-s)^{\alpha-1}-(t-s)^{\alpha-1}\right] S_{\alpha}(t-s) f\left(s, y_{s}+\widehat{\phi}_{s}\right) \mathrm{d} s\right\|^{2} \\
& +22 E\left\|\int_{0}^{t-\epsilon}\left[(t+h-s)^{\alpha-1}-(t-s)^{\alpha-1}\right] S_{\alpha}(t-s) \sigma(s) \mathrm{d} W(s)\right\|^{2} \\
& +22 E\left\|\int_{t-\epsilon}^{t}\left[(t+h-s)^{\alpha-1}-(t-s)^{\alpha-1}\right] S_{\alpha}(t-s) \sigma(s) \mathrm{d} W(s)\right\|^{2}
\end{aligned}
$$

Applying Lemma 2.12 and the Holder inequality, we obtain

$$
\begin{aligned}
& E\|\bar{z}(t+h)-\bar{z}(t)\|^{2} \leq 22\left\|T_{\alpha}(t+h)-T_{\alpha}(t)\right\|^{2}\|(-g(y+\widehat{\phi})-h(0, \phi))\|^{2} \\
& +22 E\left\|h\left(t+h, y_{t+h}+\widehat{\phi}_{t+h}\right)-h\left(t, y_{t}+\widehat{\phi}_{t}\right)\right\|^{2} \\
& +22 \frac{C_{1}(\alpha, \beta) h^{\alpha \beta}}{\alpha \beta} \int_{t}^{t+h}(t+h-s)^{\alpha \beta} E\left\|A^{\beta} h\left(s, y_{s}+\widehat{\phi}_{s}\right)\right\|^{2} \\
& +22\left(\frac{M \alpha}{\Gamma(\alpha+1)}\right)^{2} \frac{h^{\alpha}}{\alpha} \int_{t}^{t+h}(t+h-s)^{\alpha-1} E \| f\left(s, y_{s}+\widehat{\phi}_{s} \|^{2} \mathrm{~d} s\right. \\
& +22\left(\frac{M \alpha}{\Gamma(1+\alpha)}\right)^{2} \operatorname{Tr}(Q) \int_{t}^{t+h}(t+h-s)^{2(\alpha+1)} l_{r}(s) \mathrm{d} s \\
& +22\left(\frac{M \alpha}{\Gamma(1+\alpha)}\right)^{2} M_{B}^{2} \frac{h^{\alpha}}{\alpha} \int_{t}^{t+h}(t+h-s)^{\alpha-1} E\left\|u_{y+\widehat{\phi}}^{\lambda}(s)\right\|^{2} \mathrm{~d} s \\
& +22(t-\epsilon)^{2} \int_{0}^{t-\epsilon}(t+h-s)^{\alpha \beta-1}\left\|A^{1-\beta}\right\|^{2} \\
& \times\left\|\left[S_{\alpha}(t+h-s)-S_{\alpha}(t-s)\right]\right\|^{2} E\left\|A^{\beta} h\left(s, y_{s}+\widehat{\phi}_{s}\right)\right\|^{2} \mathrm{~d} s \\
& +22 \epsilon^{2} \int_{t-\epsilon}^{t}(t+h-s)^{\alpha \beta-1}\left\|A^{1-\beta}\right\|^{2} \\
& \times\left\|\left[S_{\alpha}(t+h-s)-S_{\alpha}(t-s)\right]\right\|^{2} E\left\|A^{\beta} h\left(s, y_{s}+\widehat{\phi}_{s}\right)\right\|^{2} \mathrm{~d} s \\
& +22 \frac{(t-\epsilon)^{\alpha}}{\alpha} M_{B}^{2} \int_{0}^{t-\epsilon} \| S_{\alpha}(t+h-s) \\
& -S_{\alpha}(t-s)\left\|^{2} E\right\| u^{\lambda}(s) \|^{2} \mathrm{~d} s \\
& +22 \frac{\epsilon^{\alpha}}{\alpha} M_{B}^{2} \int_{t-\epsilon}^{t}(t+h-s)^{\alpha-1} \| S_{\alpha}(t+h-s) \\
& -S_{\alpha}(t-s)\left\|^{2} E\right\| u_{y+\widehat{\phi}}^{\lambda}(s) \|^{2} \mathrm{~d} s \\
& +22 \frac{(t-\epsilon)^{\alpha}}{\alpha} \int_{0}^{t-\epsilon}(t+h-s)^{\alpha-1} \| S_{\alpha}(t+h-s) \\
& -S_{\alpha}(t-s)\left\|^{2} E\right\| f\left(s, y_{s}+\widehat{\phi}_{s}\right) \|^{2} \mathrm{~d} s \\
& +22 \frac{\epsilon^{\alpha}}{\alpha} \int_{t-\epsilon}^{t}(t+h-s)^{\alpha-1} \| S_{\alpha}(t+h-s) \\
& -S_{\alpha}(t-s)\left\|^{2} E\right\| f\left(s, y_{s}+\widehat{\phi}_{s}\right) \|^{2} \mathrm{~d} s \\
& +22 \operatorname{Tr}(Q) \int_{0}^{t-\epsilon}(t+h-s)^{2(\alpha-1)} \| S_{\alpha}(t+h-s)
\end{aligned}
$$




$$
\begin{aligned}
& -S_{\alpha}(t-s) \|^{2} l_{r}(s) \mathrm{d} s \\
& +22 \operatorname{Tr}(Q) \int_{t-\epsilon}^{t}(t+h-s)^{2(\alpha-1)} \| S_{\alpha}(t+h-s) \\
& -S_{\alpha}(t-s) \|^{2} l_{r}(s) \mathrm{d} s \\
& +22 C_{1}(\alpha, \beta) \frac{\epsilon^{2 \alpha(1-\beta)+1}}{2 \alpha(1-\beta)+1} \int_{0}^{t-\epsilon}\left[(t+h-s)^{\alpha-1}-(t-s)^{\alpha-1}\right]^{2} \\
& \times E\left\|A^{\beta} h\left(s, y_{s}+\widehat{\phi}_{s}\right)\right\|^{2} \mathrm{~d} s \\
& +22 C_{1}(\alpha, \beta) \frac{\epsilon^{2 \alpha(1-\beta)+1}}{2 \alpha(1-\beta)+1} \int_{t-\epsilon}^{t}\left[(t+h-s)^{\alpha-1}-(t-s)^{\alpha-1}\right]^{2} \\
& \times E\left\|A^{\beta} h\left(s, y_{s}+\widehat{\phi}_{s}\right)\right\|^{2} \mathrm{~d} s \\
& +22\left(\frac{M \alpha}{\Gamma(1+\alpha)}\right)^{2} M_{B}^{2} \int_{0}^{t-\epsilon}\left[(t+h-s)^{\alpha-1}-(t-s)^{\alpha-1}\right] \mathrm{d} s \\
& \times \int_{0}^{t-\epsilon}\left[(t+h-s)^{\alpha-1}-(t-s)^{\alpha-1}\right] E\left\|u^{\lambda}(s)\right\|^{2} \\
& +22\left(\frac{M \alpha}{\Gamma(1+\alpha)}\right)^{2} M_{B}^{2} \int_{t-\epsilon}^{t}\left[(t+h-s)^{\alpha-1}-(t-s)^{\alpha-1}\right] \mathrm{d} s \\
& \times \int_{t-\epsilon}^{t}\left[(t+h-s)^{\alpha-1}-(t-s)^{\alpha-1}\right] E\left\|u^{\lambda}(s)\right\|^{2} \\
& +22\left(\frac{M \alpha}{\Gamma(1+\alpha)}\right)^{2} \int_{0}^{t-\epsilon}\left[(t+h-s)^{\alpha-1}-(t-s)^{\alpha-1}\right] \mathrm{d} s \\
& \times \int_{0}^{t-\epsilon}\left[(t+h-s)^{\alpha-1}-(t-s)^{\alpha-1}\right] E\left\|f\left(s, y_{s}+\widehat{\phi}_{s}\right)\right\|^{2} \mathrm{~d} s \\
& +22\left(\frac{M \alpha}{\Gamma(1+\alpha)}\right)^{2} \int_{t-\epsilon}^{t}\left[(t+h-s)^{\alpha-1}-(t-s)^{\alpha-1}\right] \mathrm{d} s \\
& \times \int_{t-\epsilon}^{t}\left[(t+h-s)^{\alpha-1}-(t-s)^{\alpha-1}\right] E\left\|f\left(s, y_{s}+\widehat{\phi}_{s}\right)\right\|^{2} \mathrm{~d} s \\
& +22\left(\frac{M \alpha}{\Gamma(1+\alpha)}\right)^{2} \operatorname{Tr}(Q) \int_{0}^{t-\epsilon}\left[(t+h-s)^{\alpha-1}-(t-s)^{\alpha-1}\right]^{2} l_{r}(s) \mathrm{d} s \\
& +22\left(\frac{M \alpha}{\Gamma(1+\alpha)}\right)^{2} \operatorname{Tr}(Q) \int_{t-\epsilon}^{t}\left[(t+h-s)^{\alpha-1}-(t-s)^{\alpha-1}\right]^{2} l_{r}(s) \mathrm{d} s
\end{aligned}
$$

Therefore, for $\epsilon$ sufficiently small, and by the compactness of $T(t), S(t)$, we can verify that the right-hand side of the above inequality tends to zero as $h \rightarrow 0$. Thus $E\left\|\bar{z}_{2}(t+h)-\bar{z}_{1}(t)\right\|^{2} \rightarrow 0$ as $h \rightarrow 0$ for all $x \in \mathcal{B}_{r}$. This implies that $\Phi$ maps $\mathcal{B}_{r}$ into an equicontinuous of functions.

Step 4: Next we show that the set $V(t)=\left\{(\Phi y)(t): y \in \mathcal{B}_{r}\right\}$ is relatively compact in $\mathbb{H}$. The case $t=0$ is trivial. Let $t \in[0, T]$ be fixed and for each $\varepsilon \in(0, t)$, arbitrary $\delta>0$ and $y \in \mathcal{B}_{r}$, we define the operator $\Phi_{\varepsilon, \delta} y$ the set of $\bar{z}_{\varepsilon, \delta} \in \mathcal{B}_{b}^{0}$ such that

$$
\begin{aligned}
\bar{z}_{\varepsilon, \delta}(t)= & \int_{\delta}^{\infty} \xi_{\alpha}(\theta) T\left(t^{\alpha} \theta\right)(-g(y+\widehat{\phi})-h(0, \phi)) \mathrm{d} \theta+h\left(t-\varepsilon, y_{t-\varepsilon}+\widehat{\phi}_{t-\varepsilon}\right) \\
& +\alpha \int_{0}^{t-\varepsilon} \int_{\delta}^{\infty} \theta(t-s)^{\alpha-1} \xi_{\alpha}(\theta) A T\left((t-s)^{\alpha} \theta\right) h\left(s, y_{s}+\widehat{\phi}_{s}\right) \mathrm{d} \theta \mathrm{d} s \\
& +\alpha \int_{0}^{t-\varepsilon} \int_{\delta}^{\infty} \theta(t-s)^{\alpha-1} \xi_{\alpha}(\theta) T\left((t-s)^{\alpha} \theta\right) B u_{y+\widehat{\phi}}^{\lambda}(s) \mathrm{d} \theta \mathrm{d} s \\
& +\alpha \int_{0}^{t-\varepsilon} \int_{\delta}^{\infty} \theta(t-s)^{\alpha-1} \xi_{\alpha}(\theta) T\left((t-s)^{\alpha} \theta\right) f\left(s, y_{s}+\widehat{\phi}_{s}\right) \mathrm{d} \theta \mathrm{d} s
\end{aligned}
$$




$$
\begin{aligned}
& +\alpha \int_{0}^{t-\varepsilon} \int_{\delta}^{\infty} \theta(t-s)^{\alpha-1} \xi_{\alpha}(\theta) T\left((t-s)^{\alpha} \theta\right) \sigma(s) \mathrm{d} \theta \mathrm{d} W(s) \\
= & T_{\alpha}\left(\varepsilon^{\alpha} \delta\right) \int_{\delta}^{\infty} \xi_{\alpha}(\theta)\left(T\left(t^{\alpha} \theta\right)\right. \\
& \left.-T\left(\varepsilon^{\alpha} \delta\right)\right)(-g(y+\widehat{\phi})-h(0, \phi)) \mathrm{d} \theta+h\left(t-\varepsilon, y_{t-\varepsilon}+\widehat{\phi}_{t-\varepsilon}\right) \\
& +T\left(\varepsilon^{\alpha} \delta\right) \alpha \int_{0}^{t-\varepsilon} \int_{\delta}^{\infty} \theta(t-s)^{\alpha-1} \xi_{\alpha}(\theta) A\left(T\left((t-s)^{\alpha} \theta\right)\right. \\
& \left.-T\left(\varepsilon^{\alpha} \delta\right)\right) h\left(s, y_{s}+\widehat{\phi}_{s}\right) \mathrm{d} \theta \mathrm{d} s \\
& +T\left(\varepsilon^{\alpha} \delta\right) \alpha \int_{0}^{t-\varepsilon} \int_{\delta}^{\infty} \theta(t-s)^{\alpha-1} \xi_{\alpha}(\theta)\left(T\left((t-s)^{\alpha} \theta\right)\right. \\
& \left.-T\left(\varepsilon^{\alpha} \delta\right)\right) B u_{y+\widehat{\phi}^{\lambda}}(s) \mathrm{d} \theta \mathrm{d} s \\
& +T\left(\varepsilon^{\alpha} \delta\right) \alpha \int_{0}^{t-\varepsilon} \int_{\delta}^{\infty} \theta(t-s)^{\alpha-1} \xi_{\alpha}(\theta)\left(T\left((t-s)^{\alpha} \theta\right)\right. \\
& \left.-T\left(\varepsilon^{\alpha} \delta\right)\right) f\left(s, y_{s}+\widehat{\phi}_{s}\right) \mathrm{d} \theta \mathrm{d} s \\
& +T\left(\varepsilon^{\alpha} \delta\right) \alpha \int_{0}^{t-\varepsilon} \int_{\delta}^{\infty} \theta(t-s)^{\alpha-1} \xi_{\alpha}(\theta)\left(T \left((t-s)^{\alpha}\right.\right. \\
& \left.\left.-T\left(\varepsilon^{\alpha} \delta\right)\right) \theta\right) \sigma(s) \mathrm{d} \theta \mathrm{d} W(s) \\
=: & T\left(\varepsilon^{\alpha} \delta\right) p(t, \varepsilon)
\end{aligned}
$$

Since $T\left(\varepsilon^{\alpha} \delta\right)$ is compact and $p(t, \varepsilon)$ is is bounded on $\mathcal{B}_{r}$, the set $V_{\varepsilon}(t)=\left\{\left(\Phi_{\varepsilon, \delta} y\right)(t), y \in \mathcal{B}_{r}\right\}$ is relatively compact set in $\mathbb{H}$ for every $\varepsilon \in(0, t)$ and for all $\delta>0$. On the other hand, we have for every $y \in \mathcal{B}_{r}$, we have

$$
\begin{aligned}
E\left\|\bar{z}(t)-\bar{z}_{\varepsilon, \delta}(t)\right\|^{2} \leq & 10 E\left\|\int_{0}^{\delta} \xi_{\alpha}(\theta) T\left(t^{\alpha} \theta\right)(-g(y+\widehat{\phi})-h(0, \phi)) \mathrm{d} \theta\right\|^{2} \\
& +10 E\left\|h\left(t-\varepsilon, y_{t-\varepsilon}+\widehat{\phi}_{t-\varepsilon}\right)-h\left(t, y_{t}+\widehat{\phi}_{t}\right)\right\|^{2} \\
& +10 \alpha^{2} E\left\|\int_{0}^{t} \int_{0}^{\delta} \theta(t-s)^{\alpha-1} \xi_{\alpha}(\theta) A T\left((t-s)^{\alpha} \theta\right) h\left(s, y_{s}+\widehat{\phi}_{s}\right) \mathrm{d} \theta \mathrm{d} s\right\|^{2} \\
& +10 \alpha^{2} E\left\|\int_{t-\varepsilon}^{t} \int_{\delta}^{\infty} \theta(t-s)^{\alpha-1} \xi_{\alpha}(\theta) A T\left((t-s)^{\alpha} \theta\right) h\left(s, y_{s}+\widehat{\phi}_{s}\right) \mathrm{d} \theta \mathrm{d} s\right\|^{2} \\
& +10 \alpha^{2} E\left\|\int_{0}^{t} \int_{0}^{\delta} \theta(t-s)^{\alpha-1} \xi_{\alpha}(\theta) T\left((t-s)^{\alpha} \theta\right) B u_{y+\widehat{\phi}}^{\lambda}(s) \mathrm{d} \theta \mathrm{d} s\right\|^{2} \\
& +10 \alpha^{2} E\left\|\int_{t-\varepsilon}^{t} \int_{\delta}^{\infty} \theta(t-s)^{\alpha-1} \xi_{\alpha}(\theta) T\left((t-s)^{\alpha} \theta\right) B u_{y+\widehat{\phi}}^{\lambda}(s) \mathrm{d} \theta \mathrm{d} s\right\|^{2} \\
& +10 \alpha^{2} E\left\|\int_{0}^{t} \int_{0}^{\delta} \theta(t-s)^{\alpha-1} \xi_{\alpha}(\theta) T\left((t-s)^{\alpha} \theta\right) f\left(s, y_{s}+\widehat{\phi}_{s}\right) \mathrm{d} \theta \mathrm{d} s\right\|^{2} \\
& +10 \alpha^{2} E\left\|\int_{t-\varepsilon}^{t} \int_{\delta}^{\infty} \theta(t-s)^{\alpha-1} \xi_{\alpha}(\theta) T\left((t-s)^{\alpha} \theta\right) f\left(s, y_{s}+\widehat{\phi}_{s}\right) \mathrm{d} \theta \mathrm{d} s\right\|^{2} \\
& +10 \alpha^{2} E\left\|\int_{0}^{t} \int_{0}^{\delta} \theta(t-s)^{\alpha-1} \xi_{\alpha}(\theta) T\left((t-s)^{\alpha} \theta\right) \sigma(s) \mathrm{d} \theta \mathrm{d} W(s)\right\|^{2} \\
& +10 \alpha^{2} E\left\|\int_{t-\varepsilon}^{t} \int_{\delta}^{\infty} \theta(t-s)^{\alpha-1} \xi_{\alpha}(\theta) T\left((t-s)^{\alpha} \theta\right) \sigma(s) \mathrm{d} \theta \mathrm{d} W(s)\right\|^{2} \\
:= & \sum \sum_{i=1} J_{i}
\end{aligned}
$$


where

$$
\begin{aligned}
J_{1} & \leq 20 M^{2}\left(M_{g}\|y+\widehat{\phi}\|^{2}+M_{h}\left\|A^{-\beta}\right\|^{2}\left(1+\|\phi\|^{2}\right)\right)\left(\int_{0}^{\delta} \xi_{\alpha}(\theta) \mathrm{d} \theta\right)^{2} \\
J_{2} & \leq 10 M_{h}\left\|A^{-\beta}\right\|^{2}\left(\varepsilon+\left\|y_{t-\varepsilon}-y_{t}+\widehat{\phi}_{t-\varepsilon}-\widehat{\phi}_{t}\right\|^{2}\right) \\
J_{3} & \leq 10 \alpha^{2} M_{1-\beta}^{2} \int_{0}^{t}(t-s)^{\alpha \beta-1} \mathrm{~d} s \int_{0}^{t}(t-s)^{\alpha \beta-1} E\left\|A^{\beta} h\left(s, y_{s}+\widehat{\phi}_{s}\right)\right\|^{2} \mathrm{~d} s\left(\int_{0}^{\delta} \theta^{2 \beta} \xi_{\alpha}(\theta) \mathrm{d} \theta\right)^{2} \\
& \leq 10\left(\frac{\alpha M_{1-\beta} T^{\alpha \beta}}{\alpha \beta}\right)^{2} M_{h}\left(1+r^{\prime}\right)\left(\int_{0}^{\delta} \theta^{2 \beta} \xi_{\alpha}(\theta) \mathrm{d} \theta\right)^{2} \\
J_{4} & \leq 10 \alpha^{2} M_{1-\beta}^{2} \int_{t-\varepsilon}^{t}(t-s)^{\alpha \beta-1} \mathrm{~d} s \int_{t-\varepsilon}^{t}(t-s)^{\alpha \beta-1} E\left\|A^{\beta} h\left(s, y_{s}+\widehat{\phi}_{s}\right)\right\|^{2} \mathrm{~d} s\left(\int_{\delta}^{\infty} \theta^{2 \beta} \xi_{\alpha}(\theta) \mathrm{d} \theta\right)^{2} \\
& \leq 10\left(\frac{\alpha M_{1-\beta}}{\alpha \beta}\right)^{2} \varepsilon^{2 \alpha \beta} M_{h}\left(1+r^{\prime}\right)\left(\int_{\delta}^{\infty} \theta^{2 \beta} \xi_{\alpha}(\theta) \mathrm{d} \theta\right)^{2} \\
J_{5} & \leq 10\left(\alpha M M_{B}\right)^{2} \int_{0}^{t}(t-s)^{\alpha-1} \int_{0}^{t}(t-s)^{\alpha-1} E\left\|u_{y+\widehat{\phi}^{\lambda}}(s)\right\|^{2} \mathrm{~d} s\left(\int_{0}^{\delta} \theta \xi_{\alpha}(\theta) \mathrm{d} \theta\right)^{2} \\
& \leq 10\left(M M_{B}\right)^{2} T^{2 \alpha} \frac{7}{\lambda^{2}}\left(\frac{\alpha M M_{B}}{\Gamma(1+\alpha)}\right)^{2} \Delta\left(\int_{0}^{\delta} \theta \xi_{\alpha}(\theta) \mathrm{d} \theta\right)^{2} \\
J_{6} & \leq 10\left(\alpha M M_{B}\right)^{2} \int_{t-\varepsilon}^{t}(t-s)^{\alpha-1} \int_{t-\varepsilon}^{t}(t-s)^{\alpha-1} E\left\|u_{y+\widehat{\phi}}^{\lambda}(s)\right\|^{2} \mathrm{~d} s\left(\int_{\delta}^{\infty} \theta \xi_{\alpha}(\theta) \mathrm{d} \theta\right)^{2} \\
J_{9} & \leq 10 \alpha^{2} M^{2} T r(Q) \int_{0}^{t}(t-s)^{2(\alpha-1)} l_{r}(s) \mathrm{d} s\left(\int_{0}^{\delta} \theta \xi_{\alpha}(\theta) \mathrm{d} \theta\right)^{2} \\
J_{10} & \leq 10 \alpha^{2} M^{2} T r(Q) \int_{t-\varepsilon}^{t}(t-s)^{2(\alpha-1)} l_{r}(s) \mathrm{d} s\left(\int_{\delta}^{\infty} \theta \xi_{\alpha}(\theta) \mathrm{d} \theta\right)^{2} \\
J_{7} & \left.\leq 10 M^{2} M_{f}\left(1+\| M_{s}\right)^{2} \varepsilon^{2 \alpha} \frac{7}{\lambda^{2}}\left(\frac{\alpha M M_{B}}{\Gamma(1+\alpha)}\right)^{2} \Delta\left(\int_{\delta}^{\infty} \theta \xi_{\alpha}(\theta) \mathrm{d} \theta\right)^{2} \|^{2}\right) T^{2 \alpha}\left(\int_{0}^{\delta} \theta \xi_{\alpha}(\theta) \mathrm{d} \theta\right) \\
& \leq 10 M_{f}\left(1+\left\|y_{s}+\widehat{\phi}_{s}\right\|^{2}\right) \varepsilon^{2 \alpha}\left(\int_{\delta}^{\infty} \theta \xi_{\alpha}(\theta) \mathrm{d} \theta\right)^{2} \\
& \\
J^{2} &
\end{aligned}
$$

From (3.17) to (3.26), it can be easily seen that $J_{1}-J_{10}$ tends to zero as $\varepsilon \rightarrow 0$ and $\delta \rightarrow 0$. Thus, for each $y \in \mathcal{B}_{r}$

$$
E\left\|\bar{z}(t)-\bar{z}_{\varepsilon, \delta}(t)\right\|^{2} \rightarrow 0 \quad \text { as } \quad \varepsilon \rightarrow 0, \quad \delta \rightarrow 0
$$

Therefore, there are relative compact sets arbitrarily close to the set $V(t), t>0$. Hence, the set $V(t)$, $t>0$ is also relatively compact in $\mathbb{H}$.

Step 5: $\Phi$ has a closed graph.

Let $y^{n} \rightarrow y^{*}$, as $n \rightarrow \infty, \bar{z}^{n} \in \Phi y^{n}$ for each $y^{n} \in \mathcal{B}_{r}$. and $\bar{z}^{n} \rightarrow \bar{z}^{*}$ as $n \rightarrow \infty$. We shall show that $\bar{z}^{*} \in \Phi y^{*}$. Since $\bar{z}^{n} \in \Phi y^{n}$, then there exists $\sigma^{n} \in S_{G, y^{n}}$ such that 


$$
\bar{z}^{n}(t)=\left\{\begin{array}{l}
0, t \in(-\infty, 0] \\
T_{\alpha}(t)\left(-g\left(y^{n}+\widehat{\phi}\right)-h(0, \phi)\right)+h\left(t, y_{t}^{n}+\widehat{\phi}_{t}\right) \\
\quad+\int_{0}^{t}(t-s)^{\alpha-1} A S_{\alpha}(t-s) h\left(s, y_{s}^{n}+\widehat{\phi}_{s}\right) \mathrm{d} s \\
\quad+\int_{0}^{t}(t-s)^{\alpha-1} S_{\alpha}(t-s) B u_{y+\widehat{\phi}^{\lambda}}(s) \mathrm{d} s \\
\quad+\int_{0}^{t}(t-s)^{\alpha-1} S_{\alpha}(t-s) f\left(s, y_{s}^{n}+\widehat{\phi}_{s}\right) \mathrm{d} s \\
\quad+\int_{0}^{t}(t-s)^{\alpha-1} S_{\alpha}(t-s) \sigma^{n}(s) \mathrm{d} W(s), \quad t \in J .
\end{array}\right.
$$

We must prove that there exists $\sigma^{*} \in S_{G, y^{*}}$ such that

$$
\bar{z}^{*}(t)=\left\{\begin{array}{l}
0, \quad t \in(-\infty, 0] \\
T_{\alpha}(t)\left(-g\left(y^{*}+\widehat{\phi}\right)-h(0, \phi)\right)+h\left(t, y_{t}^{*}+\widehat{\phi}_{t}\right) \\
\quad+\int_{0}^{t}(t-s)^{\alpha-1} A S_{\alpha}(t-s) h\left(s, y_{s}^{*}+\widehat{\phi}_{s}\right) \mathrm{d} s \\
\quad+\int_{0}^{t}(t-s)^{\alpha-1} S_{\alpha}(t-s) B u_{y+\widehat{\phi}^{\lambda}}(s) \mathrm{d} s \\
\quad+\int_{0}^{t}(t-s)^{\alpha-1} S_{\alpha}(t-s) f\left(s, y_{s}^{*}+\widehat{\phi}_{s}\right) \mathrm{d} s \\
\quad+\int_{0}^{t}(t-s)^{\alpha-1} S_{\alpha}(t-s) \sigma^{*}(s) \mathrm{d} W(s), \quad t \in J .
\end{array}\right.
$$

Now, for every $t \in J$, since $h$ is continuous, and from the definition of $u^{\lambda}$ we get

$$
\begin{aligned}
E \| & \left(\bar{z}^{n}(t)-T_{\alpha}(t)\left(-g\left(y^{n}+\widehat{\phi}\right)-h(0, \phi)\right)-h\left(t, y_{t}^{n}+\widehat{\phi}_{t}\right)\right. \\
& -\int_{0}^{t}(t-s)^{\alpha-1} A S_{\alpha}(t-s) h\left(s, y_{s}^{n}+\widehat{\phi}_{s}\right) \mathrm{d} s-\int_{0}^{t}(t-s)^{\alpha-1} S_{\alpha}(t-s) B u_{y+\widehat{\phi}}^{\lambda}(s) \mathrm{d} s \\
& \left.-\int_{0}^{t}(t-s)^{\alpha-1} S_{\alpha}(t-s) f\left(s, y_{s}^{n}+\widehat{\phi}_{s}\right) \mathrm{d} s\right) \\
& -\left(\bar{z}^{*}(t)-T_{\alpha}(t)\left(-g\left(y^{*}+\widehat{\phi}\right)-h(0, \phi)\right)-h\left(t, y_{t}^{*}+\widehat{\phi}_{t}\right)\right. \\
& +\int_{0}^{t}(t-s)^{\alpha-1} A S_{\alpha}(t-s) h\left(s, y_{s}^{*}+\widehat{\phi}_{s}\right) \mathrm{d} s-\int_{0}^{t}(t-s)^{\alpha-1} S_{\alpha}(t-s) B u_{y+\widehat{\phi}}^{\lambda}(s) \mathrm{d} s \\
& \left.-\int_{0}^{t}(t-s)^{\alpha-1} S_{\alpha}(t-s) f\left(s, y_{s}^{*}+\widehat{\phi}_{s}\right) \mathrm{d} s\right) \|^{2} \\
\rightarrow & 0 \text { as } n \rightarrow \infty
\end{aligned}
$$

Consider the linear continuous operator $(\Theta \sigma)(t): L^{2}(J, \mathbb{H}) \rightarrow \mathcal{C}(J, \mathbb{H})$

$$
\begin{aligned}
\sigma \mapsto(\Theta \sigma)(t)= & \int_{0}^{t}(t-s)^{\alpha-1} S_{\alpha}(t-s) \sigma(s) \mathrm{d} W(s) \\
& -\int_{0}^{t}(t-s)^{\alpha-1} S_{\alpha}(t-s) B B^{*} S_{\alpha}^{*}(T-t) \\
& \times\left(\int_{0}^{T}\left(\lambda I+\Gamma_{s}^{T}\right)^{-1}(T-s)^{\alpha-1} S_{\alpha}(T-s) \sigma(s) \mathrm{d} W(s)\right) \mathrm{d} s
\end{aligned}
$$


We can see that the operator $\Theta$ is linear and continuous. Moreover, on has

$$
\|\Theta \sigma\|^{2} \leq\left(\frac{M T^{\alpha}}{\Gamma(1+\alpha)}\right)^{2} \operatorname{Tr}(Q)\|\sigma\|_{L^{2}(\mathbb{U}, \mathbb{H})}^{2}\left[2+\left(\frac{M M_{B} T^{\alpha}}{\Gamma(1+\alpha)}\right)^{2} \frac{2}{\lambda^{2}}\left(\frac{\alpha M M_{B}}{\Gamma(1+\alpha)}\right)^{2}\right]
$$

From Lemma 2.9, it follows that $\Theta \circ S_{G}$ is a closed graph operator. Also, from the definition of $\Theta$, we have that

$$
\begin{aligned}
& \left(\bar{z}^{n}(t)-T_{\alpha}(t)\left(-g\left(y^{n}+\widehat{\phi}\right)-h(0, \phi)\right)-h\left(t, y_{t}^{n}+\widehat{\phi}_{t}\right)\right. \\
& -\int_{0}^{t}(t-s)^{\alpha-1} A S_{\alpha}(t-s) h\left(s, y_{s}^{n}+\widehat{\phi}_{s}\right) \mathrm{d} s-\int_{0}^{t}(t-s)^{\alpha-1} S_{\alpha}(t-s) B u_{y+\widehat{\phi}^{\lambda}}^{\lambda}(s) \mathrm{d} s \\
& \left.\quad-\int_{0}^{t}(t-s)^{\alpha-1} S_{\alpha}(t-s) f\left(s, y_{s}^{n}+\widehat{\phi}_{s}\right) \mathrm{d} s\right) \in \Theta\left(S_{G, y^{n}}\right)
\end{aligned}
$$

Since $y^{n} \rightarrow y^{*}$, for some $y^{*} \in S_{G, x}$, it follows from Lemma 2.9 that

$$
\begin{aligned}
& \left(\bar{z}^{*}(t)-T_{\alpha}(t)\left(-g\left(y^{*}+\widehat{\phi}\right)-h(0, \phi)\right)-h\left(t, y_{t}^{*}+\widehat{\phi}_{t}\right)\right. \\
& +\int_{0}^{t}(t-s)^{\alpha-1} A S_{\alpha}(t-s) h\left(s, y_{s}^{*}+\widehat{\phi}_{s}\right) \mathrm{d} s-\int_{0}^{t}(t-s)^{\alpha-1} S_{\alpha}(t-s) B u_{y+\widehat{\phi}}^{\lambda}(s) \mathrm{d} s \\
& \left.-\int_{0}^{t}(t-s)^{\alpha-1} S_{\alpha}(t-s) f\left(s, y_{s}^{*}+\widehat{\phi}_{s}\right) \mathrm{d} s\right) \in \Theta\left(S_{G, y^{n}}\right)
\end{aligned}
$$

therefore $\Phi$ has a closed graph.

As a consequence of step 1 to step 5 with the Arzela-Ascoli theorem, we conclude that $\Phi$ is a compact multivalued map, u.s.c. with convex closed values. As a consequence of Lemma 2.13, we can deduce that $\Phi$ has a fixed point $x($.$) on \mathcal{B}_{r}$, which is a mild solution of system (2.1).

\section{Approximate controllability of fractional stochastic control systems}

In this section, we present our main result on approximate controllability of system, to prove this, the following additional assumption is required;

(H6) The linear fractional inclusion (3.1) is approximately controllable.

(H7) The functions $h: J \times \mathcal{B}_{h} \rightarrow \mathbb{H}_{\beta}, f: J \times \mathcal{B}_{h} \rightarrow \mathbb{H}$ and $G: J \times \mathcal{B}_{h} \rightarrow B C C(L(\mathbb{U}, \mathbb{H}))$ are uniformly bounded for all $t \in J$ and $x \in \mathcal{B}_{h}$

Theorem 4.1 Assume that the assumptions of Theorem 3.4 hold, and in addition, hypothesis (H6) and (H7) are satisfied. Then, the fractional stochastic differential inclusion (2.1) is approximately controllable on $J$.

Proof Let $x^{\lambda}(.) \in \mathcal{B}_{r}$ be a fixed point of the operator $\Phi$. By Theorem 3.4, any fixed point of $\Phi$ is a mild solution of (2.1). This means that there is $x^{\lambda} \in \Phi\left(x^{\lambda}\right)$, that is, by the stochastic Fubini theorem, there is $\sigma^{\lambda} \in S_{G, x^{\lambda}}$ such that, 


$$
\begin{aligned}
x_{x}^{\lambda}(t)= & \bar{x}_{T}-\lambda\left(\lambda I+\Gamma_{0}^{T}\right)^{-1}\left[E \bar{x}_{T}+\int_{0}^{t} \eta(s) \mathrm{d} W(s)+T_{\alpha}(T)(\phi(0)\right. \\
& \left.\left.-g\left(x^{\lambda}\right)-h(0, \phi)\right)-h\left(T, x_{T}^{\lambda}\right)\right] \\
& +\lambda \int_{0}^{t}\left(\lambda I+\Gamma_{s}^{T}\right)^{-1}(T-s)^{\alpha-1} A S_{\alpha}(T-t) h\left(s, x_{s}^{\lambda}\right) \mathrm{d} s \\
& +\lambda \int_{0}^{t}\left(\lambda I+\Gamma_{s}^{T}\right)^{-1}(T-s)^{\alpha-1} S_{\alpha}(T-t) f\left(s, x_{s}^{\lambda}\right) \mathrm{d} s \\
& +\lambda \int_{0}^{t}\left(\lambda I+\Gamma_{s}^{T}\right)^{-1}(T-s)^{\alpha-1} S_{\alpha}(T-t) \sigma^{\lambda}(s) \mathrm{d} W(s)
\end{aligned}
$$

By the conditions (H2), we can choose a sufficiently small positive constant $\epsilon>0, \alpha+\epsilon<1$, such that $A^{\alpha+\epsilon} h\left(T, x_{T}^{\lambda}\right)$ is bounded in $\mathbb{H}$. Since

$$
h\left(T, x_{T}^{\lambda}\right)=A^{-(\alpha+\epsilon)} A^{\alpha+\epsilon} h\left(T, x_{T}^{\lambda}\right),
$$

we see that $h\left(T, x_{T}^{\lambda}\right)$ is relatively compact in $\mathbb{H}$, so there is $x^{1} \in \mathbb{H}$ such that (by passing to a subsequence)

$$
h\left(T, x_{T}^{\lambda}\right) \rightarrow x^{1}
$$

in $\|$.$\| as \lambda \rightarrow 0^{+}$

Moreover, by the boundedness of $h, f$ and $G$ (assumption (H7)) and Dunford-Pettis Theorem, we have that the sequences $\left\{\sigma^{\lambda}(s)\right\},\left\{A^{\beta} h\left(s, x_{s}^{\lambda}\right\}\right.$ and $\left\{f\left(s, x_{s}^{\lambda}\right)\right\}$ are weakly compact in $L^{2}(J, L(\mathbb{U}, \mathbb{H})), L^{2}\left([0, T], \mathbb{H}_{\beta}\right)$ and $L^{2}([0, T], \mathbb{H})$, so there are a subsequences still denoted by $\left\{\sigma^{\lambda}(s)\right\},\left\{A^{\beta} h\left(s, x_{s}^{\lambda}\right\}\right.$ and $\left\{f\left(s, x_{s}^{\lambda}\right)\right\}$, that weakly converge to, say, $\sigma, h$ and $f$ respectively in $L^{2}(J, L(\mathbb{U}, \mathbb{H})), L^{2}\left([0, T], \mathbb{H}_{\beta}\right)$ and $L^{2}([0, T], \mathbb{H})$.

On the other hand, by assumption $(H 6)$, the operator $\lambda\left(\lambda I+\Gamma_{s}^{T}\right)^{-1} \longrightarrow 0$ strongly as $\lambda \longrightarrow 0^{+}$for all $0 \leq s \leq T$, and, moreover, $\left\|\lambda\left(\lambda I+\Gamma_{s}^{T}\right)^{-1}\right\| \leq 1$. Thus, the Lebesgue dominated convergence theorem and the compactness of $S_{\alpha}(t)$ yield

$$
\begin{aligned}
E\left\|x^{\lambda}(t)-\bar{x}_{T}\right\|^{2} \leq & 10\left\|\lambda\left(\lambda I+\Gamma_{0}^{T}\right)^{-1}\right\|^{2} E\left\|\left[E \bar{x}_{T}+T_{\alpha}(T)\left(\phi(0)-g\left(x^{\lambda}\right)-h(0, \phi)\right)\right]\right\|^{2} \\
& +10\left\|\lambda\left(\lambda I+\Gamma_{0}^{T}\right)^{-1}\left(h\left(T, x_{T}^{\lambda}\right)-x^{1}\right)\right\|^{2}+10\left\|\lambda\left(\lambda I+\Gamma_{0}^{T}\right)^{-1} x^{1}\right\|^{2} \\
& +10 E\left\|\lambda\left(\lambda I+\Gamma_{0}^{T}\right)^{-1} \int_{0}^{t} \eta(s) \mathrm{d} W(s)\right\|^{2} \\
& +10 E\left\|\int_{0}^{t} \lambda\left(\lambda I+\Gamma_{s}^{T}\right)^{-1}(T-s)^{\alpha-1} A S_{\alpha}(T-t)\left(h\left(s, x_{s}^{\lambda}\right)-h(s)\right) \mathrm{d} s\right\|^{2} \\
& +10 E\left\|\int_{0}^{t} \lambda\left(\lambda I+\Gamma_{s}^{T}\right)^{-1}(T-s)^{\alpha-1} A S_{\alpha}(T-t) h(s) \mathrm{d} s\right\|^{2} \\
& +10 E\left\|\int_{0}^{t} \lambda\left(\lambda I+\Gamma_{s}^{T}\right)^{-1}(T-s)^{\alpha-1} S_{\alpha}(T-t)\left(f\left(s, x_{s}^{\lambda}\right)-f(s)\right) \mathrm{d} s\right\|^{2} \\
& +10 E\left\|\int_{0}^{t} \lambda\left(\lambda I+\Gamma_{s}^{T}\right)^{-1}(T-s)^{\alpha-1} S_{\alpha}(T-t) f(s) \mathrm{d} s\right\|^{2} \\
& +10 E \int_{0}^{t}\left\|\lambda\left(\lambda I+\Gamma_{s}^{T}\right)^{-1}(T-s)^{\alpha-1} S_{\alpha}(T-t)\left(\sigma^{\lambda}(s)-\sigma(s)\right)\right\|_{L^{2}(\mathbb{U}, \mathbb{H})}^{2} \mathrm{~d} s \\
& +10 E \int_{0}^{t}\left\|\lambda\left(\lambda I+\Gamma_{s}^{T}\right)^{-1}(T-s)^{\alpha-1} S_{\alpha}(T-t) \sigma(s)\right\|_{L^{2}(\mathbb{U}, \mathbb{H})}^{2} \mathrm{~d} s \longrightarrow 0, \\
& \quad \text { as } \lambda \rightarrow 0^{+}
\end{aligned}
$$

So $x^{\lambda}(t) \rightarrow \bar{x}_{T}$ holds, which shows that the system (2.1) is is approximately controllable and the proof is complete. 
Remark 4.2 We notice that, in the case of infinite-dimensional systems, we can distinguish two concepts of controllability: Exact and approximate controllability. Exact controllability means that the system can be steered to an arbitrary final state. Approximate controllability enables us to steer the system to an arbitrary small neighborhood of the final state. In the finite-dimensional case, the notions of approximate and exact controllability coincide. Moreover, Approximate controllable systems are more prevalent and very often approximate controllability is completely adequate in applications (see [37,38]). However, the problems of exact controllability are developed in numerous papers. Ren et al. [36] studied the controllability of a class of impulsive neutral stochastic functional differential inclusions with infinite delay in an abstract space. Sufficient conditions for the controllability are derived with the help of the fixed point theorem for discontinuous multivalued operators due to Dhage [15]. Li and Zou [23] obtained sufficient conditions for the controllability of nonlinear neutral stochastic differential inclusions with infinite delay in a Hilbert space with using a fixed-point theorem for condensing maps due to ORegan [29]. Li and Peng [24], Ganesh Priya and Muthukumar [31] studied the controllability of a class of fractional stochastic functional differential systems. Based on these works, the exact controllability of the system 2.1 can be done by relying on a fixed-point theorem for condensing maps due to ORegan [29] and employing the idea and technique as in Theorem 8 in [23].

\section{An example}

As an application, we consider a stochastic partial differential inclusion with the following form

$$
\left\{\begin{array}{l}
D_{t}^{\alpha}\left[v(t, x)-\int_{-\infty}^{t} e^{4(t-s)} v(s, x) \mathrm{d} s\right] \in \frac{\partial^{2}}{\partial x^{2}} v(t, x) \\
\left.+b(x) u(t)+\int_{-\infty}^{t} a_{1}(s-t) \sin v(s, x)\right) \mathrm{d} s \\
\quad+\int_{-\infty}^{t} a_{2}(s-t) v(s, x) \mathrm{d} s \mathrm{~d} \beta(t), x \in[0, \pi], t \in J=[0, T], \quad T>0, \\
v(t, 0)=v(t, \pi)=0 \quad t \in[0, T] \\
v(0, x)+\int_{0}^{\pi} \xi(x, z) v(t, z) \mathrm{d} z=\varphi(t, x), \quad t \in(-\infty, 0], \quad 0 \leq x \leq \pi, .
\end{array}\right.
$$

where $\beta(t)$ is a standard cylindrical Wiener process in $\mathbb{H}$ defined on a stochastic space $\left(\Omega, \mathcal{F},\left\{\mathcal{F}_{t}\right\}_{t \geq 0}, \mathbb{P}\right)$; the fractional derivative $D_{t}^{\alpha}, 0<\alpha<1$ is understood in the Caputo sense; the functions $\xi \in C([0, \pi] \times[0, \pi], \mathbb{R})$ and $a_{1}, a_{2}$ are continues.

Let $\mathbb{U}=\mathbb{H}=L^{2}([0, \pi])$ with the norm $\|$.$\| . Now, we present a special phase space \mathcal{B}_{h}$. Let $h(t)=e^{2 t}$, $t<0$, Then $l=\int_{-\infty}^{0} h(s) \mathrm{d} s=\frac{1}{2}$. Let

$$
\|\varphi\|_{\mathcal{B}_{h}}=\int_{-\infty}^{0} h(s) \sup _{s \leq \theta \leq 0}\left(E\|\varphi(\theta)\|^{2}\right)^{\frac{1}{2}}
$$

then $\left(\mathcal{B}_{h},\|\cdot\|_{\mathcal{B}_{h}}\right)$ is a Banach space.

Define an infinite-dimensional space $\mathbb{U}$ by $\mathbb{U}=\left\{u / u=\sum_{n=2}^{\infty} u_{n} w_{n}\right.$ with $\left.\sum_{n=2}^{\infty} U_{n}^{2}<\infty\right\}$. The norm in $\mathbb{U}$ is defined by $\|u\|_{\mathbb{U}}^{2}=\sum_{n=2}^{\infty} U_{n}^{2}$. Now, define a continuous linear mapping $B$ from $\mathbb{U}$ into $\mathbb{H}$ as $B u=$ $2 u_{2} w_{1}+\sum_{n=2}^{\infty} u_{n} w_{n}$ for $u=\sum_{n=2}^{\infty} u_{n} w_{n} \in \mathbb{U}$.

We define the operator $A$ by $A x=\frac{\partial^{2} x}{\partial y^{2}}$. with domain

$$
D(A)=\left\{x \in \mathbb{H}, \frac{\partial x}{\partial y}, \frac{\partial^{2} x}{\partial y^{2}} \in \mathbb{H} \text { and } x(0)=x(\pi)=0\right\}
$$

It is well known that $\mathrm{A}$ generates an analytic semigroup $T(t), t \geq 0$ given by

$$
T(t) x=\sum_{n=1}^{\infty} e^{-n^{2} t}\left\langle x, e_{n}\right\rangle e_{n},
$$

$x \in H$, and $e_{n}(y)=(2 / \pi)^{1 / 2} \sin (n y), n=1,2, \ldots$, is the orthogonal set of eigenvectors of $A$. 
For $(t, \varphi) \in J \times \mathcal{B}_{h}$, where $\varphi(\theta)(x)=\varphi(\theta, x),(\theta, x) \in(-\infty, 0] \times[0, \pi]$. Let $v(t)()=v(t, x)$, and define the bounded linear operator $B: \mathbb{U} \rightarrow \mathbb{H}$ by $B u(t)(x)=b(t) u(x), 0 \leq x \leq \pi$.

Define the Lipschitz continuous functions $h: J \times \mathcal{B}_{h} \rightarrow L^{2}([0, \pi]), f: J \times \mathcal{B}_{h} \rightarrow L^{2}([0, \pi])$ and $G: J \times \mathcal{B}_{h} \rightarrow L\left(L^{2}([0, \pi]), L^{2}([0, \pi])\right)$ by

$$
\begin{aligned}
h(t, \varphi)(x) & =\int_{-\infty}^{0} e^{4(\theta)}(\varphi(\theta)(x)) \mathrm{d} \theta, \\
f(t, \varphi) & =\int_{-\infty}^{0} a_{1}(\theta)(\sin \varphi(\theta)(x)) \mathrm{d} \theta, \\
G(t, \varphi) & =\int_{-\infty}^{0} a_{2}(\theta)(\varphi(\theta)(x)) \mathrm{d} \theta .
\end{aligned}
$$

Then, the system (5.1) can be rewritten as the abstract form of system (2.1). Thus, under the appropriate conditions on the functions $h, f, G$ and $g$ as those in (H1)-(H6), system (5.1) has a mild solution and is approximately controllable on $J$.

\section{Concluding remarks}

In this paper, we have investigated the approximate controllability of class of fractional neutral stochastic evolution inclusion with nonlocal initial conditions in Hilbert space. Based on a fixed-point theorem, sufficient conditions for the existence of solutions and the approximate controllability of fractional nonlinear stochastic differential inclusions have been derived. Impulsive fractional differential equations and inclusions have become important in recent years as mathematical models of many phenomena in both physical and social sciences [38]. For the basic theory of impulsive differential equations and inclusions the reader can refer to [5,21]. Recently, Debbouche and Baleanu [13] established the controllability result for a class of fractional evolution nonlocal impulsive quasilinear delay integro-differential systems in a Banach space by using the theory of fractional calculus and fixed point technique. More recently, Liu and Li [22] established the controllability of impulsive fractional evolution differential inclusions with initial boundary conditions in Banach spaces by applying the fixed point theorem for multivalued maps due to Dhage association with an evolution system. Upon making some appropriate assumption on system functions, by adapting the techniques and ideas established in the paper of [13] and [22] with suitable modifications, one can prove the existence of solutions and the approximate controllability of fractional stochastic differential inclusions with nonlocal condition and impulses of the form:

$$
\left\{\begin{array}{l}
{ }^{c} D_{t}^{\alpha}\left[x(t)-h\left(t, x_{t}\right)\right] \in A x(t)+B u(t)+f\left(t, x_{t}\right) \\
\quad+G\left(t, x_{t}\right) \frac{\mathrm{d} W(t)}{\mathrm{d} t}, \quad t \in J=[0, T], \quad T>0, \quad t \neq t_{k} \\
\Delta x\left(t_{k}\right)=I_{k}\left(x\left(t_{k}^{-}\right)\right)=x\left(t_{k}^{+}\right)-x\left(t_{k}^{-}\right), \quad k=1, \ldots, m \\
x(0)+g(x)=x_{0}=\phi, \quad \phi \in \mathcal{B}_{h} .
\end{array}\right.
$$

In our future work we will investigate the existence and controllability results of fractional stochastic differential inclusions driven by fractional Brownian motion.

Acknowledgements The authors would like to thank the referee for valuable comments and suggestions.

Open Access This article is distributed under the terms of the Creative Commons Attribution 4.0 International License (http:// creativecommons.org/licenses/by/4.0/), which permits unrestricted use, distribution, and reproduction in any medium, provided you give appropriate credit to the original author(s) and the source, provide a link to the Creative Commons license, and indicate if changes were made.

\section{References}

1. Agarwal, R.P.; Bashir, A.; Alsaedi, A.; Shahzad, N.: On the dimension of the solution set for semilinear fractional differential inclusions. Abstr. Appl. Anal. 2012, 10, Art ID 305924 (2012). doi:10.1155/2012/305924

2. Benchohra, M.; Ziane, M.: Impulsive evolution inclusions with state-dependent delay and multivalued jumps. Electron. J. Qual. Theory Differ. Equ. 42, 1-21 (2013) 
3. Balasubramaniam, P.; Park, J.Y.; Vincent Antony Kumar, A.: Existence of solutions for semilinear neutral stochastic functional differential equations with nonlocal conditions. Nonlinear Anal. TMA 71, 1049-1058 (2009)

4. Balasubramaniam, P.; Vembarasan, V.; Senthilkumar, T.: Approximate controllability of impulsive fractional integrodifferential systems with nonlocal conditions in Hilbert Space. Numer. Funct. Anal. Optim. 35, 177-197 (2014)

5. Benchohra, M.; Henderson, J.; Ntouyas, N.: Impulsive Differential Equations and Inclusions, vol. 2. Hindawi Publishing Corporation, New York (2006)

6. Bohnenblust, H.F.; Karlin, S.: On a theorem of Ville. In: Kuhn, H.W., Tucker, A.W. (eds.) Contributions to the Theory of Games. Annals of Mathematics Studies, vol. 1, pp. 155-160. Princeton University Press, Princeton (1950)

7. Boudaoui, A.; Slama, A.: Approximate controllability of nonlinear fractional impulsive stochastic differential equations with nonlocal conditions and infinite delay. Nonlinear Dyn. Syst. Theory 16(1), 35-48 (2016)

8. Byszewski, L.; Lakshmikantham, V.: Theorem about the existence and uniqueness of solutions of a nonlocal Cauchy problem in a Banach space. Appl. Anal. 40, 11-19 (1990)

9. Byszewski, L.: Theorems about the existence and uniqueness of solutions of a semilinear evolution nonlocal Cauchy problem. J. Math. Anal. Appl. 162, 494-506 (1991)

10. Byszewski, L.: Existence and uniqueness of solutions of semilinear evolution nonlocal Cauchy problem. Zesz. Nauk. Politech. Rzesz. Mat. Fiz. 18, 109-112 (1993)

11. Chadha, A.; Pandey, D.N.: Existence of the mild solution for impulsive neutral stochastic fractional integro-differential inclusions with nonlocal conditions. Mediterr. J. Math. 13(3), 1005-1031 (2016)

12. Caputo, M.: Elasticit e Dissipazione. Zanichelli, Bologna (1969)

13. Debbouche, A.; Baleanu, D.: Controllability of fractional evolution nonlocal impulsive quasilinear delay integro-differential systems. Comput. Math. Appl. 62, 1442-1450 (2011)

14. Deimling, K.: Multivalued Differential Equations. De Gruyter, Berlin (1992)

15. Dhage, B.C.: Fixed point theorems for discontinuous multi-valued operators on ordered spaces with applications. Comput. Math. Appl. 51, 589-604 (2006)

16. El-Sayed, A.M.A.; Ibrahim, A.G.: Multivalued fractional differential equations of arbitrary orders. Appl. Math. Comput. 68, $15-25$ (1995)

17. Guendouzi, T.; Bousmaha, L.: Approximate controllability of fractional neutral stochastic functional integro-differential inclusions with infinite delay. Qual. Theory Dyn. Syst. (2014). doi:10.1007/s12346-014-0107-y

18. Hu, S.; Papageorgiou, N.: Handbook of Multivalued Analysis. Kluwer, Dordrecht (1997)

19. Kilbas, A.A.; Srivastava, H.M.; Trujillo, J.J.: Theory and Applications of Fractional Differential Equations. Elsevier Science B.V, Amsterdam (2006)

20. Lasota, A.; Opial, Z.: An application of the Kakutani-Ky Fan theorem in the theory of ordinary differential equations. Bull. Acad. Pol. Sci. Ser. Sci. Math. Astron. Phys. 13, 781-786 (1965)

21. Lakshmikantham, V.; Bainov, D.D.; Simeonov, P.S.: Theory of Impulsive Differential Equations. World Scientific, Singapore (1989)

22. Liu, Z.; Li, X.: On the controllability of impulsive fractional evolution inclusions in Banach space. J. Optim. Theory Appl. 156, 167-182 (2013). doi:10.1007/s10957-012-0236-X

23. Li, Y., Zou, Q.: Controllability of nonlinear neutral stochastic differential inclusions with infinite delay. Math. Probl. Eng. 2013, 13, Art ID 419156 (2013). doi:10.1155/2013/419156

24. Li, K.; Peng, J.: Controllability of fractional neutral stochastic functional differential systems. Zeitschrift fr angewandte Mathematik und Physik 65(5), 941-959 (2014)

25. Mahmudov, N.I.: Controllability of linear stochastic systems in Hilbert spaces. J. Math. Anal. Appl. 259, 64-82 (2001)

26. Mahmudov, N.I.: Approximate controllability of semilinear deterministic and stochastic evolution equations in abstract spaces. SIAM J. Control Optim. 42, 1604-1622 (2003)

27. Mahmudov, I.N.; Denker, A.: On controllability of linear stochastic systems. Int. J. Control 73, 144-151 (2000)

28. Miller, K.S.; Ross, B.: An Introduction to the Fractional Calculus and Differential Equations. Wiley, New York (1993)

29. O'Regan, D.: Nonlinear alternatives for multivalued maps with applications to operator inclusions in abstract spaces. Proc. Am. Math. Soc. 127(12), 3557-3564 (1999)

30. Podlubny, I.: Fractional Differential Equations. Academic, San Diego (1999)

31. Priya, B.G.; Muthukumar, P.: Controllability study on fractional order impulsive stochastic differential equation. IFACPapersOnLine 49(1), 516-521 (2016)

32. Rajivganthi, C.; Thiagu, K.; Muthukumar, P.; Balasubramaniam, P.: Dindigul: Existence of solutions and approximate controllability of impulsive fractional stochastic differential systems with infinite delay and Poisson jumps. Appl. Math. 60(4), 395-419 (2015)

33. Rajivganthi, C.; Muthukumar, P.; Ganesh Priya, B.: Approximate controllability of fractional stochastic integro-differential equations with infinite delay of order $1<\alpha<2$. IMA J. Math. Control Inf. 1-15 (2015). doi:10.1093/imamci/dnv005

34. Ren, Y.; Zhou, Q.; Chen, L.: Existence, uniqueness and stability of mild solutions for time-dependent stochastic evolution equations with Poisson jumps and infinite delay. J. Optim. Theory Appl. 149, 315-331 (2011)

35. Ren, Y.; Sun, D.D.: Second-order neutral stochastic evolution equations with infinite delay under Caratheodory conditions. J. Optim. Theory Appl. 147, 569-582 (2010)

36. Ren, Y.; Hu, L.; Sakthivel, R.: Controllability of impulsive neutral stochastic functional differential inclusions with infinite delay. J. Comput. Appl. Math. 235, 26032614 (2011)

37. Sakthivel, R.; Nieto, J.J.; Mahmudov, N.I.: Approximate controllability of nonli- near deterministic and stochastic systems with unbounded delay. Taiwan. J. Math. 14(5), 1777-1797 (2010)

38. Sakthivel, R.; Ganesh, R.; Anthoni, S.M.: Approximate controllability of fractional nonlinear differential inclusions. Appl. Math. Comput. 225, 708-717 (2013)

39. Sakthivel, R.; Ren, Y.; Debbouche, A.; Mahmudov, N.I.: Approximate controllability of fractional stochastic differential inclusions with nonlocal conditions. Appl. Anal. (2015). doi:10.1080/00036811.2015.1090562 
40. Slama, A.; Boudaoui, A.: Existence of solutions for nonlinear fractional impulsive stochastic differential equations with nonlocal conditions and infinite delay. Int. J. Differ. Equ. Appl. 13(4), 185-201 (2014)

41. Wang, J.; Zhou, Y.: Existence and controllability results for fractional semilinear differentail inclusions. Nonlinear Anal.: RWA 12, 3642-3653 (2011)

42. Yan, Z.: On a nonlocal problem for fractional integrodifferential inclusions in Banach spaces. Ann. Polon. Math. 101, 87-104 (2011)

43. Yan, Z.; Jia, X.: Impulsive problems for fractional partial neutral functional integro-differential inclusions with infinite delay and analytic resolvent operators. Mediterr. J. Math. (2013). doi:10.1007/s00009-013-0349-y

44. Yan, Z.; Jia, X.: Approximate controllability of partial fractional neutral stochastic functional integro-differential inclusions with state-dependent delay. Collectanea Mathematica. University of Barcelona (2014). doi:10.1007/s13348-014-0109-8

45. Yan, Z.; Lu, F.: On approximate controllability of fractional stochastic neutral integro-differential inclusions with infinite delay. Appl. Anal. Int. J. (2014). doi:10.1080/00036811.2014.924214

46. Zhou, Y.; Jiao, F.: Existence of mild solution for fractional neutral evolution equations. Comput. Math. Appl. 59, 1063-1077 (2010) 